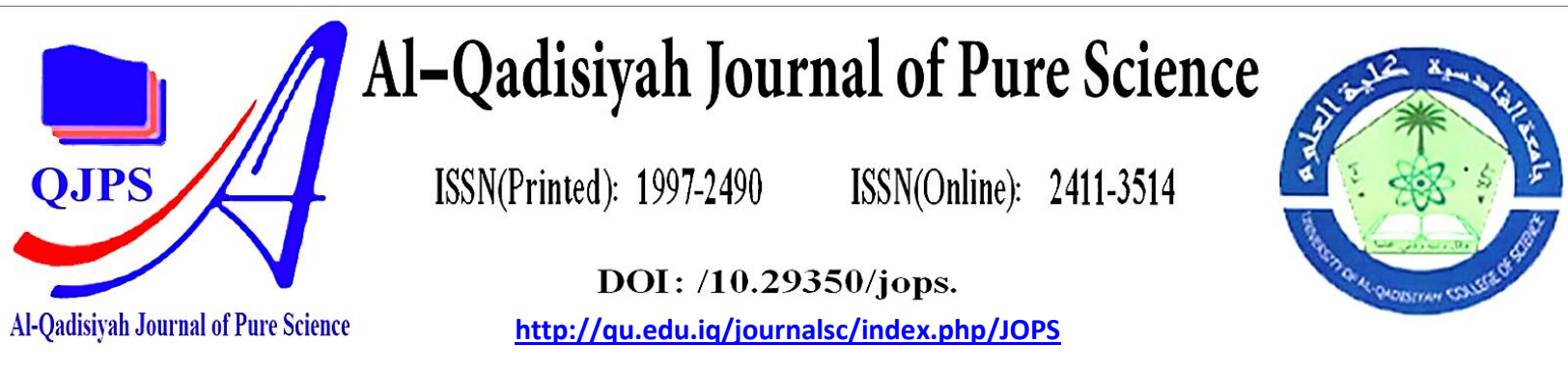

\title{
Magnetohydrodynamics Peristaltic Flow of a Couple- Stress with Varying Temperature and concentration for Jeffrey Fluid through a Flexible Porous Medium
}

\author{
Authors Names \\ a. Saif Razzaq Mohsion \\ Al-Waily \\ b. Dheia G. Salih \\ Al-Khafajy
}

\section{Article History}

Received on: $19 / 6 / 2021$

Revised on: $20 / 7 / 2021$

Accepted on:25/7/2021

\section{Keywords:}

Jeffrey fluid, couple-stress flow, porous channel, wall properties.

DOI: https://doi.org/10.29350/

jops.2021.26. 4.1373

\section{ABSTRACT}

The topic of this paper is the peristaltic motion of a non-Newtonian Jeffrey fluid with couple stress across a porous medium inside a horizontal conduit. The unit is strained by a uniform magnetic field. It is taken into account the effects of viscous dissipation, internal heat generation, and radiation. This approach solves the equations of momentum, temperature, and velocity. The numerical formulas for temperature, axial velocity, and velocity are calculated as functions of the problem's physical parameters. Numerical calculations, as well as the effects of temperature and the inclined slanted magnetic field and concentration on the velocity equation, were conducted for this formula, and the results were shown on the channel wall. The results of the problem's physical parameters In a series of statistics, the effects of this formula are explained numerically and graphically.

\section{Intoduction}

Peristaltic fluxes have piqued the interest of scientists. many rasearchers due to their weide Physiology and industry applications. The peristaltic system motility of blood in animals or human bodies has been studied by several authors. Peristaltic pumping has been found in many applications. Several researchers presented their scientific findings in the search for peristaltic flow of different flow geometries and were among the pioneersin this field to Latham[7]. In[10] and [8], they presented a comprehensive study of peristaltic flow fluid together with experimental results in which For this, the long wave approximation was used the analysis In a circular cylindrical tube, there is a peristaltic pumping problem. Furthermore, peristalsis exposed to magnetic field impacts is critical in the treatment of hyperthermia, arterial flow, cancer treatment, and other conditions. etc.; magnets can cause infections, ulcers, intestines and uterine diseases. Permeability has a big role and it has important applications in fluid movement, for example absorption of food in the intestine, extraction of oil from the ground, etc. Several researchers are involved in See [3], [5], [9], and [11] for research on the combined effect of a magnetic field and the presence of permeability in a fluid flow channel. The influence of temperature on the movement of fluids through a channel sparked interest, as most studies agreed that increasing the temperature increases the fluid's velocity The velocity of the fluid, on the other hand, fluctuates in an ambiguous manner in response to the concentration difference. For more information, see [4], [1], and [2]

\footnotetext{
a Department of Mathematics, College of Science, University of Al-Qadisiyah, Diwaniyah, Iraq, E-Mail: saif.r.m.1993@gmail.com

${ }^{\mathrm{b}}$ Department of Mathematics, College of Education for Girls, University of Al-Kufa, Najaf, Iraq, E-Mail:

dr.dheia.g.salih@gmail.com, dheia.salih@qu.edu.iq
} 
depending on where the fluid is in the channel. Hoseinzadeh and colleagues. [6] investigated the impact of the modified Darcy law on fluid flow in porous walls channels. The present analysis aims to discuss the effects of MHD on the couplestrains of Jeffrey fluid through a medium porous cylindrical channel. To our knowledge, this attempt has not yet been explored in the presence of the magnetic field, the effect of temperature difference, and the double pressure concentration of a Jeffrey fluid flow in cylindrical coordinates through a porous channel.

\section{Mathematical Formulation}

Considering of flow a peristaltic a couple stress the incompressible Jeffrey fluid with the inclined slanted magnetic field and temperature with concentration of a circular pipe that is coaxial and uniform. The Jeffrey fluid is a non-Newtonian non-compressible fluid model that represents the real fluid when shear stress does not equal shear stress rate (or velocity gradient). The cylindrical coordinates are known when $\mathrm{R}$ is along the channel's radius and $\mathrm{Z}$ is parallel to the pipe's axes, as shown in figure 1.

$\mathcal{L}$

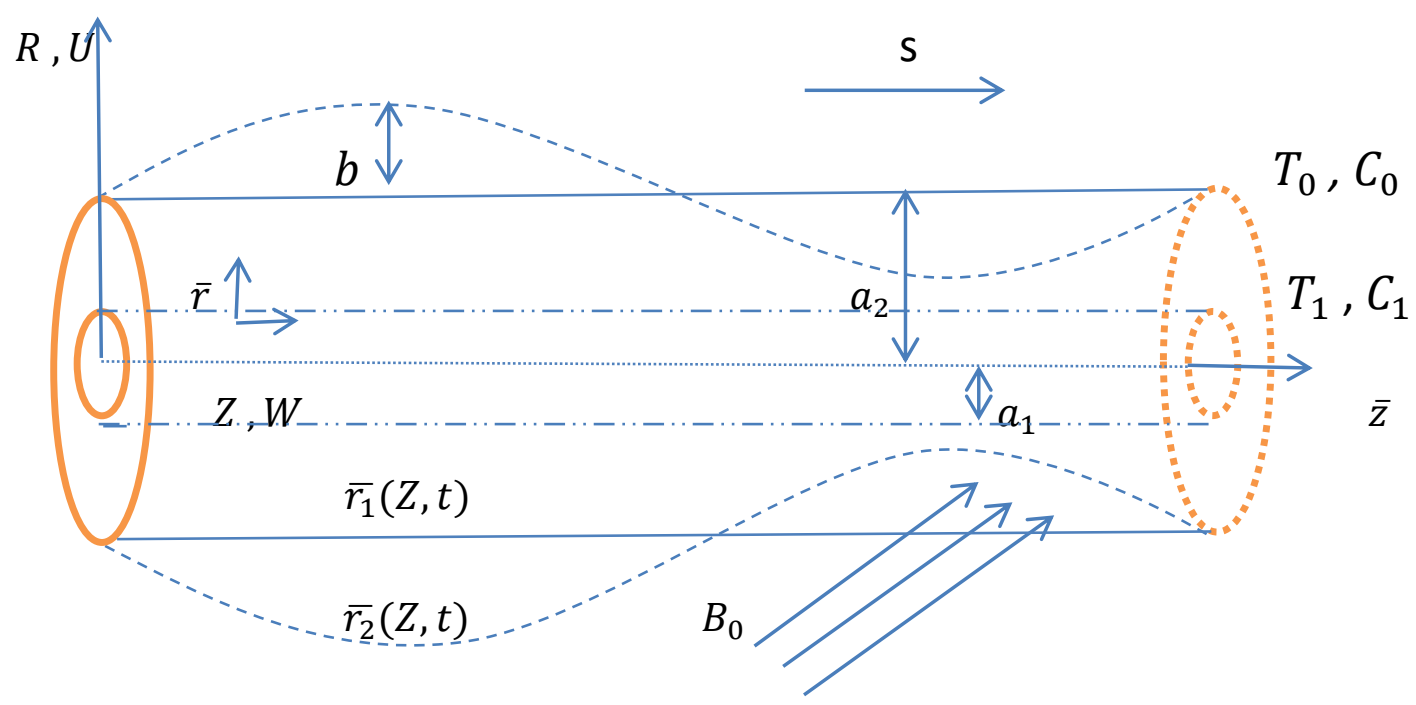

Figure. 1: The problem's geometry

The following is a description of the wall surface geometry:

$$
\left.\begin{array}{ll}
\bar{r}=\overline{r_{1}}=a_{1} & \text { Inner wall } \\
\bar{r}=\bar{r}_{2}(\bar{z}, \bar{t})=a_{2}+b \operatorname{Sin}\left(\frac{2 \pi}{\mathcal{L}}(\bar{z}-s \bar{t})\right) & \text { Outer wall }
\end{array}\right\}
$$

Where $a_{1}$ is now the average radius of a channel that hasn't been affected, $a_{2}$ is now the average radius of a disturbed channel, $b$ was its the peristaltic wave's amplitude, $\mathcal{L}$ was itswavelength, $\mathrm{s}$ was its speed of wave propagation, and $\bar{t}$ isthe time

\section{Basic Equations}

The governing equations are as follows:

The equation of continuity; $\quad \nabla \bar{V}=0$,

The equation of momentum with couple-stress fluid it's given by;

$\rho(\bar{V} \cdot \nabla) \bar{V}=\nabla \bar{\sigma}+\mu_{p} \bar{J} \times \bar{B}-\frac{\mu}{K^{*}} \bar{V}+\rho g \beta_{1}\left(T-T_{0}\right)+\rho g \beta_{2}\left(C-C_{0}\right)-\bar{\zeta} \bar{\nabla}^{4} \bar{V}$, 
The equation of temperature it's given by;

$T_{s} \cdot \rho(\bar{V} \cdot \nabla) T=T_{c} \cdot \nabla^{2} T-\nabla \cdot Q_{r}-q\left(T-T_{0}\right)$,

The equation of concentration is given in:

$$
(\bar{V} . \nabla) C=D_{m} \nabla^{2} C+\frac{D_{m} T_{d}}{T_{m}} \nabla^{2} T .
$$

When $\nabla^{2}=\frac{1}{r} \frac{\partial}{\partial r}\left(r \frac{\partial}{\partial r}\right)$ is the "Laplace operator", $\nabla^{4}=\nabla^{2}\left(\nabla^{2}\right)$. Also $\bar{V}$ is the velocity field, $\rho$ "density", $\mu$ "dynamic viscosity" dependent on temperature, $k^{*}$ "permeability", we observe that the magnetic field's effect is appear on the flow in the $\mathrm{R}$ and $\mathrm{Z}$ direction, $\mathrm{J}$ "induced current", $\bar{B}=\left(0, B_{0} \sin (\varpi), 0\right)$ the inclined magnetic field, $\mu_{p}$ "magnetic permeability", $\bar{\sigma}$ "Cauchy stress tensor" and $\bar{\zeta}$ is constant related to the couple stress. Also $T$ and $C$ are "temperature and concentration", $T_{c}$ "thermal conductivity", $T_{S}$ "specific heat capacity" at constant pressure, $D_{m}$ "coefficient of mass diffusivity", $\nabla V$ the "fluid velocity gradient", $T_{m}$ "mean fluid temperature", $Q_{r}$ "radiation heat flux", $q$ "heat generation" and $T_{d}$ "thermal diffusion ratio".

For an incompressible Jeffrey fluid, the constitutive equations are as follows;

$\bar{\sigma}=-\bar{P} \bar{I}+\bar{S}$,

$\bar{S}=\frac{\mu}{1+\lambda_{1}}\left(\bar{\gamma}+\lambda_{2} \bar{\gamma}\right)$.

where $\bar{S}$ "extra stress tensor", $\bar{P}$ "pressure", $\bar{I}$ "identity tensor", $\lambda_{1}$ "ratio of relaxation to retardation times", $\overline{\dot{\gamma}}$ " shear ratio", $\bar{\gamma}$ "material derivative", and $\lambda_{2}$ "retardation time".

\section{Method of solution}

The two-frame coordinates transformations public and private are given by;

$\bar{r}=\bar{R}, \bar{z}=\bar{Z}, \bar{u}=\bar{U}, \bar{w}=\bar{W}-s$.

where $(\bar{u}, \bar{w})$ and $(\bar{U}, \bar{W})$ are the velocity components in public and private frames, respectively. The equations of motion are solved using these transforms;

$\frac{\partial \bar{u}}{\partial \bar{r}}+\frac{\bar{u}}{\bar{r}}+\frac{\partial(\overline{\mathrm{w}}+\mathrm{s})}{\partial \bar{z}}=0$,

$\rho\left(\frac{\partial \bar{u}}{\partial \bar{t}}+\bar{u} \frac{\partial \bar{u}}{\partial \bar{r}}+(\bar{w}+s) \frac{\partial \bar{u}}{\partial \bar{z}}\right)=-\frac{\partial \bar{p}}{\partial \bar{r}}+\frac{1}{\bar{r}} \frac{\partial}{\partial \bar{r}}\left(\bar{r} \bar{S}_{\bar{r} \bar{r}}\right)+\frac{\partial}{\partial \bar{z}}\left(\bar{S}_{\bar{r} \bar{z}}\right)-\frac{\bar{S}_{\bar{\vartheta} \bar{\vartheta}}}{\bar{r}}-\sigma\left(B_{0} \operatorname{Sin}(\varpi)\right)^{2} \bar{u}-$ $\frac{\mu}{k^{*}} \bar{u}-\bar{\zeta} \bar{\nabla}^{4} \bar{u}$

$\rho\left(\frac{\partial(\overline{\mathrm{w}}+\mathrm{s})}{\partial \overline{\mathrm{t}}}+\overline{\mathrm{u}} \frac{\partial(\overline{\mathrm{w}}+\mathrm{s})}{\partial \overline{\mathrm{r}}}+(\overline{\mathrm{w}}+\mathrm{s}) \frac{\partial(\overline{\mathrm{w}}+\mathrm{s})}{\partial \overline{\mathrm{z}}}\right)=-\frac{\partial \overline{\mathrm{p}}}{\partial \overline{\mathrm{z}}}+\frac{1}{\overline{\mathrm{r}}} \frac{\partial}{\partial \overline{\mathrm{r}}}\left(\overline{\mathrm{r}} \bar{S}_{\overline{\mathrm{r}} \overline{\mathrm{z}}}\right)+\frac{\partial}{\partial \overline{\mathrm{z}}}\left(\bar{S}_{\overline{\mathrm{z}} \overline{\mathrm{z}}}\right)+\rho g \beta_{1}(T-$

$\left.T_{0}\right)+\rho g \beta_{2}\left(C-C_{0}\right)-\sigma\left(B_{0} \operatorname{Sin}(\varpi)\right)^{2}(\bar{w}+s)-\frac{\mu}{\mathrm{k}^{*}}(\overline{\mathrm{w}}+\mathrm{s})-\bar{\zeta} \bar{\nabla}^{4}(\overline{\mathrm{w}}+\mathrm{s})$,

$\frac{\partial \mathrm{T}}{\partial \overline{\mathrm{t}}}+\bar{u} \frac{\partial T}{\partial \bar{r}}+(\bar{W}+s) \frac{\partial T}{\partial \bar{z}}=\frac{T_{C}}{T_{S} \rho}\left(\frac{\partial^{2} T}{\partial \bar{r}^{2}}+\frac{1}{\bar{r}} \frac{\partial T}{\partial \bar{r}}+\frac{\partial^{2} T}{\partial \bar{z}^{2}}\right)-\frac{16 \sigma_{0} T_{2}^{E}}{3 k_{0} T_{S} \rho} \frac{1}{\bar{r}} \frac{\partial}{\partial \bar{r}}\left(\bar{r} \frac{\partial T}{\partial \bar{r}}\right)-\frac{q}{T_{S} \rho}\left(T-T_{0}\right)$,

$\frac{\partial C}{\partial \bar{t}}+\bar{u} \frac{\partial C}{\partial \bar{r}}+(\bar{w}+s) \frac{\partial C}{\partial \bar{z}}=D_{m}\left(\frac{\partial^{2} C}{\partial \bar{r}^{2}}+\frac{1}{\bar{r}} \frac{\partial C}{\partial \bar{r}}+\frac{\partial^{2} C}{\partial \bar{z}^{2}}\right)+\frac{D_{m} T_{d}}{T_{m}}\left(\frac{\partial^{2} T}{\partial \bar{r}^{2}}+\frac{1}{\bar{r}} \frac{\partial T}{\partial \bar{r}}+\frac{\partial^{2} T}{\partial \bar{z}^{2}}\right)$.

The relevant boundary conditions are:

$\left.\begin{array}{l}\bar{w}=-1, \bar{u}=0, T=T_{1}, C=C_{1} \text { at } \bar{r}=r_{1}=a_{1}, \\ \bar{w}=-1, \bar{u}=0, T=T_{0}, C=C_{0} \text { at } \bar{r}=\bar{r}_{2}(\bar{z}, \bar{t})=a_{2}+b \operatorname{Sin}\left(\frac{2 \pi}{\mathcal{L}}(\bar{z}-s \bar{t})\right) .\end{array}\right\}$

The governing equation for a flexible wall channel properties [5], is obtained as;

$L^{*}\left(\overline{r_{2}}\right)=p-p_{0}=\left(A \frac{\partial^{4}}{\partial \bar{Z}^{4}}-N \frac{\partial^{2}}{\partial \bar{Z}^{2}}+m \frac{\partial^{2}}{\partial \bar{t}^{2}}+C \frac{\partial}{\partial \bar{t}}+K_{L}\right)\left(\overline{r_{2}}\right)$ 
where $A$ is the wall's flexural rigidity, $N$ is the longitudinal tension per unit width, $m$ is the mass perunit area, $C$ is the coefficientof viscous damping and $K_{L}$ is spring stiffness and $p_{0}$ is the pressure on the wall's outside surface caused by muscle tension.

The following dimensionless transformations were conferred in order to simplify the motion's governing equations;

$$
\begin{aligned}
& u=\frac{\bar{u} \mathcal{L}}{a_{2} s}, w=\frac{\bar{w}}{s}, r=\frac{\bar{r}}{a_{2}}, \quad z=\frac{\bar{z}}{\mathcal{L}}, S=\frac{a_{2} \bar{s}}{\mu s}, \delta=\frac{a_{2}}{\mathcal{L}}, D a=\frac{k}{a_{2}^{2}}, \\
& \mathcal{H}=\frac{T-T_{0}}{T_{1}-T_{0}}, C=\frac{C-C_{0}}{C_{1}-C_{0}}, R n=\frac{K_{0} T_{s} \mu}{4 T_{2}^{E} \sigma_{0}}, p=\frac{a_{2}^{2} \bar{p}}{\mu S \mathcal{L}}, M_{1}^{2}=\frac{\sigma a_{2}^{2} B_{0}^{2}}{\mu} \sin ^{2}(\varpi), \\
& r_{1}=\frac{\overline{r_{1}}}{a_{2}}=\varepsilon<1, r_{2}=\frac{\overline{r_{2}}}{a_{2}}=1+\emptyset \sin (2 \pi \bar{z}), \alpha=\bar{\alpha} a_{2}=\sqrt{\frac{\mu}{\zeta}} a_{2} \text {, } \\
& \operatorname{Pr}=\frac{\mu T_{s}}{T_{c}}, \Omega=\frac{q a_{2}^{2}}{\mu T_{s}}, S r=\frac{\rho D_{m} T_{d}\left(T_{1}-T_{0}\right)}{\mu T_{m}\left(C_{1}-C_{0}\right)}, S c=\frac{\mu}{\rho D_{m}}, \quad t=\frac{s \bar{t}}{\mathcal{L}}, \\
& G r=\frac{\rho g \beta_{1} a_{2}^{2}\left(T_{1}-T_{0}\right)}{\mu s}, G c=\frac{\rho g \beta_{2} a_{2}^{2}\left(C_{1}-C_{0}\right)}{\mu s}, \varnothing=\frac{b}{a_{2}}, R e=\frac{\rho s a_{2}}{\mu}, \bar{\nabla}=\frac{\nabla}{a_{2}} .
\end{aligned}
$$

where $\varnothing$ "amplitude ratio", $\bar{\alpha}$ "couple stress" The ratio of the tube radius (constant) to the material characteristic length is a fluid parameter. $(\sqrt{\mu / \zeta}$, has the dimensionof length $), R e$ "Reynolds number", $P r$ "Prandtl number", $D a$ "Darcy number", $R n$ "thermal radiation parameter", $S c$ "Schmidt number", $S r$ "Soret number", $G r$ "thermal Grashof number", $G c$ "Solutal Grashof number", $M_{1}^{2}$ "magnetic parameter", $\delta$ "dimensionless wave number" and $\Omega$ "heat source/sink parameter".

Introducing non-dimensional analysis (15) for equations (8)-(14) then dropping over-bars, the pervious governing equations and boundary conditions can be rewritten in the following form;

$\left(\frac{s}{\mathcal{L}}\right)\left(\frac{\partial u}{\partial r}+\frac{u}{r}+\frac{\partial w}{\partial z}\right)=0$

$\operatorname{Re} \delta^{3}\left(\frac{\partial u}{\partial t}+u \frac{\partial u}{\partial r}+(w+1) \frac{\partial u}{\partial z}\right)=-\frac{\partial p}{\partial r}+\delta \frac{1}{r} \frac{\partial}{\partial r}\left(r S_{r r}\right)+\delta^{2} \frac{\partial}{\partial z}\left(S_{r z}\right)-\delta \frac{S_{\vartheta \vartheta}}{r}-\frac{\delta^{2}}{D a} u-$ $\frac{\delta^{2}}{\alpha^{2}} \nabla^{4} u-\delta^{2} M_{1}^{2} u$,

$\operatorname{Re} \delta\left(\frac{\partial \mathrm{w}}{\partial \mathrm{t}}+u \frac{\partial w}{\partial r}+(w+1) \frac{\partial w}{\partial z}\right)=-\frac{\partial p}{\partial z}+\frac{1}{r} S_{r z}+\frac{\partial}{\partial r}\left(S_{r z}\right)+\delta \frac{\partial}{\partial z}\left(S_{z z}\right)-\left(M_{1}^{2}+\right.$

$\left.\frac{1}{D_{a}}\right) w+G r \mathcal{H}+G c C-\left(M_{1}^{2}+\frac{1}{D_{a}}\right)-\frac{1}{\alpha^{2}} \nabla^{4}(\mathrm{w}+1)$,

$\operatorname{Re} \delta\left(\frac{\partial T}{\partial t}+u \frac{\partial \mathcal{H}}{\partial r}+(w+1) \frac{\partial \mathcal{H}}{\partial z}\right)=\frac{1}{P r}\left(\frac{\partial^{2} \mathcal{H}}{\partial r^{2}}+\frac{1}{r} \frac{\partial \mathcal{H}}{\partial r}+\delta^{2} \frac{\partial^{2} \mathcal{H}}{\partial z^{2}}\right)-\frac{4}{3 R n} \frac{1}{r} \frac{\partial}{\partial r}\left(r \frac{\partial \mathcal{H}}{\partial r}\right)-\Omega \mathcal{H}$,

$\delta \operatorname{Re}\left(\frac{\partial \mathcal{C}}{\partial t}+u \frac{\partial C}{\partial r}+(w+1) \frac{\partial C}{\partial z}\right)=\frac{1}{S c}\left(\frac{\partial^{2} C}{\partial r^{2}}+\frac{1}{r} \frac{\partial C}{\partial r}+\delta^{2} \frac{\partial^{2} C}{\partial z^{2}}\right)+S r\left(\frac{\partial^{2} \mathcal{H}}{\partial r^{2}}+\frac{1}{r} \frac{\partial \mathcal{H}}{\partial r}+\delta^{2} \frac{\partial^{2} \mathcal{H}}{\partial z^{2}}\right)$.

Where

$$
\begin{aligned}
& S_{r r}=\frac{2 \delta}{1+\lambda_{1}}\left[1+\frac{s \lambda_{2} \delta}{a_{2}}\left(\frac{\partial}{\partial t}+u \frac{\partial}{\partial r}+(w+1) \frac{\partial}{\partial z}\right)\right]\left(\frac{\partial u}{\partial r}\right) \\
& S_{r z}=\frac{1}{1+\lambda_{1}}\left[1+\frac{s \lambda_{2} \delta}{a_{2}}\left(\frac{\partial}{\partial t}+u \frac{\partial}{\partial r}+(w+1) \frac{\partial}{\partial z}\right)\right]\left(\frac{\partial w}{\partial r}+\delta^{2} \frac{\partial u}{\partial z}\right) \\
& S_{\vartheta \vartheta}=\frac{2 \delta}{1+\lambda_{1}}\left[\frac{u}{r}+\frac{s \lambda_{2} \delta}{a_{2}}\left(\frac{1}{r} \frac{\partial u}{\partial t}+\frac{u}{r} \frac{\partial u}{\partial r}-\frac{u^{2}}{r^{2}}+(w+1) \frac{1}{r} \frac{\partial u}{\partial z}\right)\right] \\
& S_{z z}=\frac{2 \delta}{1+\lambda_{1}}\left[1+\frac{s \lambda_{2} \delta}{a_{2}}\left(\frac{\partial}{\partial t}+u \frac{\partial}{\partial r}+(w+1) \frac{\partial}{\partial z}\right)\right]\left(\frac{\partial w}{\partial z}\right)
\end{aligned}
$$


with corresponding dimensional boundary conditions are;

$\begin{array}{rlc}w=-1, u=0, C=1, \mathcal{H}=1 & \text { at } & r=r_{1}=\varepsilon \\ w=-1, u=0, C=0, \mathcal{H}=0 & \text { at } & r=r_{2}=1+\emptyset \operatorname{Sin}(2 \pi(z-t)) .\end{array}$

Continuity of stress means that the pressureat the fluid-wall interface must be the same as the pressure acting on the fluid at $r=r_{2}$. The dynamic boundary conditions at the complaint walls are obtained using the $z$ momentum equation;

$L_{1} \frac{\partial^{5}(\mathrm{~h})}{\partial \mathrm{z}^{5}}-L_{2} \frac{\partial^{3}(\mathrm{~h})}{\partial \mathrm{z}^{3}}+L_{3} \frac{\partial^{3}(\mathrm{~h})}{\partial \mathrm{z} \partial \mathrm{t}^{2}}+L_{4} \frac{\partial^{2}(\mathrm{~h})}{\partial \mathrm{z} \partial \mathrm{t}}+L_{5} \frac{\partial(\mathrm{h})}{\partial \mathrm{z}}=\frac{1}{\mathrm{r}} \mathrm{S}_{\mathrm{rz}}+\frac{\partial}{\partial \mathrm{r}}\left(\mathrm{S}_{\mathrm{rz}}\right)-\left(M_{1}^{2}+\frac{1}{D_{a}}\right) w+$

$G r \mathcal{H}+G c C-\left(M_{1}^{2}+\frac{1}{D_{a}}\right)-\frac{1}{\alpha^{2}} \nabla^{4}(\mathrm{w}+1)$.

Where $L_{1}=\frac{A a_{2}^{3}}{\mu s \mathcal{L}^{5}}, L_{2}=-\frac{N a_{2}^{3}}{\mu s \mathcal{L}^{3}}, L_{3}=\frac{m s a_{2}^{3}}{\mu \mathcal{L}^{3}}, L_{4}=\frac{C a_{2}^{3}}{\mu \mathcal{L}^{2}}$, and $L_{5}=\frac{K_{L} a_{2}^{3}}{\mu s \mathcal{L}}$, whereas $L_{1}$ is the flexuralrigidity of the wall, $L_{2}$ is the per-unit-width longitudinal tension, $L_{3}$ is the mass per square meter, $L_{4}$ is the viscous damping coefficient, and $L_{5}$ is the stiffness of a spring, respectively.

Because it appears impossible to answer the problem in its generalized form, we will limit our analysis to the assumption of a tiny dimensionless wave number $(\delta \ll 1)$.

In other words, we discovered the approximate long-wavelength. Equations (16)-(26) result from this assumption:

$\frac{\partial u}{\partial r}+\frac{u}{r}+\frac{\partial w}{\partial z}=0$,

$\frac{\partial p}{\partial r}=0$,

$\frac{\partial p}{\partial z}=\frac{1}{\mathrm{r}} \mathrm{S}_{\mathrm{rz}}+\frac{\partial}{\partial \mathrm{r}}\left(\mathrm{S}_{\mathrm{rz}}\right)-\left(M_{1}^{2}+\frac{1}{D_{a}}\right) w+G r \mathcal{H}+G c \mathrm{C}-\left(M_{1}^{2}+\frac{1}{D_{a}}\right)-\frac{1}{\alpha^{2}} \nabla^{4}(\mathrm{w}+1)$,

$\left(\frac{1}{P r}-\frac{4}{3 R n}\right) \frac{1}{r} \frac{\partial}{\partial r}\left(r \frac{\partial \mathcal{H}}{\partial r}\right)-\Omega \mathcal{H}=0$,

$\frac{1}{S c}\left(\frac{\partial^{2} C}{\partial r^{2}}+\frac{1}{r} \frac{\partial C}{\partial r}\right)=-S r\left(\frac{\partial^{2} \mathcal{H}}{\partial r^{2}}+\frac{1}{r} \frac{\partial \mathcal{H}}{\partial r}\right)$,

$L_{1} \frac{\partial^{5}(\mathrm{~h})}{\partial \mathrm{z}^{5}}-L_{2} \frac{\partial^{3}(\mathrm{~h})}{\partial \mathrm{z}^{3}}+L_{3} \frac{\partial^{3}(\mathrm{~h})}{\partial \mathrm{z} \partial \mathrm{t}^{2}}+L_{4} \frac{\partial^{2}(\mathrm{~h})}{\partial \mathrm{z} \partial \mathrm{t}}+L_{5} \frac{\partial(\mathrm{h})}{\partial \mathrm{z}}=\frac{1}{\mathrm{r}} \mathrm{S}_{\mathrm{rz}}+\frac{\partial}{\partial \mathrm{r}}\left(\mathrm{S}_{\mathrm{rz}}\right)-\left(M_{1}^{2}+\frac{1}{D_{a}}\right) w+$

$G r \mathcal{H}+G c C-\left(M_{1}^{2}+\frac{1}{D_{a}}\right)-\frac{1}{\alpha^{2}} \nabla^{4}(\mathrm{w}+1)$.

Where

$S_{r r}=S_{\theta \theta}=S_{z z}=0$ and $S_{r z}=\frac{1}{1+\lambda_{1}}\left(\frac{\partial w}{\partial r}\right)$

Replecing $S_{r z}$ fromequation (33) in to equation (32), we have:

$\frac{1}{\alpha^{2}} \nabla^{4} \mathrm{w}-\frac{1}{1+\lambda_{1}} \frac{1}{r} \frac{\partial}{\partial r}\left(r \frac{\partial w}{\partial r}\right)+\left(M_{1}^{2}+\frac{1}{D a}\right) w=-\left(L_{1} \frac{\partial^{5}(\mathrm{~h})}{\partial \mathrm{z}^{5}}-L_{2} \frac{\partial^{3}(\mathrm{~h})}{\partial \mathrm{z}^{3}}+L_{3} \frac{\partial^{3}(\mathrm{~h})}{\partial \mathrm{z} \partial \mathrm{t}^{2}}+L_{4} \frac{\partial^{2}(\mathrm{~h})}{\partial \mathrm{z} \partial \mathrm{t}}+\right.$

$\left.L_{5} \frac{\partial(\mathrm{h})}{\partial \mathrm{z}}+\left(M_{1}^{2}+\frac{1}{D a}\right)-G r \mathcal{H}-G c \mathrm{C}\right)$.

We shall get the following dimensionless boundary conditions if the elements of a pair stress tensor at the wall remain zero:

$$
\begin{array}{lll}
w=-1, \frac{\partial^{2} w}{\partial r^{2}}-\frac{\alpha}{r} \frac{\partial w}{\partial r}=0 & \text { at } \quad r=\varepsilon \\
w=-1, \frac{\partial^{2} w}{\partial r^{2}}-\frac{\alpha}{r} \frac{\partial w}{\partial r}=0 & \text { at } \quad r=r_{2}
\end{array}
$$


Where $\alpha=\frac{\mu}{\zeta}$ is a couplestress fluid parameter ( $\mu$ and $\zeta$ are constantsassociated with the couplestress, when $\alpha \rightarrow 1$ (i.e. $\mu \rightarrow \zeta$ ) no couple stresseffect [9].

\section{Solutions of the Problem}

\section{1 "Temperature and Concentration" Functions}

Rewrite the temperature equation (30) as follow;

$r^{2} \frac{\partial^{2} \mathcal{H}}{\partial r^{2}}+r \frac{\partial \mathcal{H}}{\partial r}+A r^{2} \mathcal{H}=0$

where $\mathrm{A}=-\frac{3 \Omega \operatorname{Pr} R n}{3 R n-4 \operatorname{Pr}}$. Equation (36) is a zero-order modified Bessel equation. The answer to the equation(36) with boundary conditions $\mathcal{H}\left(r_{1}\right)=1, \mathcal{H}\left(r_{2}\right)=0$ is;

$\mathcal{H}=B_{1} \mathrm{~J}_{0}[r \sqrt{A}]+B_{2} \mathrm{Y}_{0}[r \sqrt{A}]$

Where $B_{1}=\frac{\mathrm{Y}_{0}[h \sqrt{A}]}{J_{0}[\epsilon \sqrt{A}] \mathrm{Y}_{0}[h \sqrt{A}]-J_{0}[h \sqrt{A}] \mathrm{Y}_{0}[\epsilon \sqrt{A}]}$ and $B_{2}=\frac{J_{0}[h \sqrt{A}]}{J_{0}[h \sqrt{A}] \mathrm{Y}_{0}[\epsilon \sqrt{A}]-J_{0}[\epsilon \sqrt{A}] \mathrm{Y}_{0}[h \sqrt{A}]}$.

The equation of concentration (31), can be written as;

$\frac{1}{r} \frac{\partial}{\partial r}\left(r \frac{\partial C}{\partial r}\right)=-S c \operatorname{Sr} \frac{1}{r} \frac{\partial}{\partial r}\left(r \frac{\partial \mathcal{H}}{\partial r}\right)$.

The general solution of equation (38) is:

$\mathrm{C}=-\operatorname{Sc} \operatorname{Sr} \mathcal{H}+B_{3} \ln (r)+B_{4}$.

Where $B_{3}=\frac{1+S_{c} S_{r}}{\operatorname{Ln}(\varepsilon /(1+\emptyset \sin (2 \pi z)))}$ and $B_{4}=-B_{3} \ln (1+\emptyset \sin (2 \pi z))$

\subsection{Momentum Function}

Rewrite the momentum equation (34) as follow;

$w=$

$B_{1} I_{0}\left(b r \sqrt{\left|s_{1}\right|}\right)+B_{2} K_{0}\left(b r \sqrt{\left|s_{1}\right|}\right)+B_{3} I_{0}\left(b r \sqrt{\left|s_{2}\right|}\right)+B_{4} K_{0}\left(b r \sqrt{\left|s_{2}\right|}\right)-$

$\frac{1}{M_{1}^{2}+\frac{1}{D_{a}}}\left(\begin{array}{c}L_{1}\left(32 \pi^{5} \emptyset \operatorname{Cos}[2 \pi(z-t)]\right)-L_{2}\left(-8 \pi^{3} \emptyset \operatorname{Cos}[2 \pi(z-t)]\right)+L_{3}\left(-8 \pi^{3} \emptyset \operatorname{Cos}[2 \pi(z-t)]\right)+ \\ L_{4}\left(4 \pi^{2} \emptyset \operatorname{Sin}[2 \pi(z-t)]\right)+L_{5}(2 \pi \emptyset \operatorname{Cos}[2 \pi(z-t)])+\left(M_{1}^{2}+\frac{1}{D_{a}}\right)-G r \mathcal{H}-G c C\end{array}\right)$.

where $b^{4}=\frac{\alpha^{2}}{D a}, s_{1}=-\frac{c_{1}}{b^{2}}-\sqrt{\left(\frac{c_{1}}{b^{2}}\right)^{2}-1}, s_{2}=-\frac{c_{1}}{b^{2}}+\sqrt{\left(\frac{c_{1}}{b^{2}}\right)^{2}-1}$ and $c_{1}=\frac{\alpha^{2}}{2\left(1+\lambda_{1}\right)}$ with condition $\frac{c_{1}}{b^{2}}>1$. Also $I_{0}, K_{0}$ are "the modified Bessel functions" of the first and second kind of zero order. By using the Mathematica12 we have a constants $B_{1}, B_{2}, B_{3}$ and $B_{4}$ in our program and boundary conditions (29).

\subsection{Stream Function}

The corresponding functions of stream $\left(w=\frac{1}{r} \frac{\partial \psi}{\partial r}\right)$ is; 


$$
\begin{aligned}
& \psi=\int r\left\{B_{1} I_{0}\left(b r \sqrt{\left|s_{1}\right|}\right)+B_{2} K_{0}\left(b r \sqrt{\left|s_{1}\right|}\right)+B_{3} I_{0}\left(b r \sqrt{\left|s_{2}\right|}\right)+B_{4} K_{0}\left(b r \sqrt{\left|s_{2}\right|}\right)-\right. \\
& \left.\frac{1}{M_{1}^{2}+\frac{1}{D_{a}}}\left(\begin{array}{c}
L_{1}\left(32 \pi^{5} \emptyset \operatorname{Cos}[2 \pi(z-t)]\right)-L_{2}\left(-8 \pi^{3} \emptyset \operatorname{Cos}[2 \pi(z-t)]\right)+L_{3}\left(-8 \pi^{3} \emptyset \operatorname{Cos}[2 \pi(z-t)]\right)+ \\
L_{4}\left(4 \pi^{2} \emptyset \operatorname{Sin}[2 \pi(z-t)]\right)+L_{5}(2 \pi \emptyset \operatorname{Cos}[2 \pi(z-t)])+\left(M_{1}^{2}+\frac{1}{D_{a}}\right)-G r \mathcal{H}-G c C
\end{array}\right)\right\} d r
\end{aligned}
$$

So that

$$
\begin{aligned}
& \psi= \\
& \frac{r^{2}}{2}\left(\begin{array}{c}
L_{1}\left(32 \pi^{5} \emptyset \operatorname{Cos}[2 \pi(z-t)]\right)-L_{2}\left(-8 \pi^{3} \emptyset \cos [2 \pi(z-t)]\right)+L_{3}\left(-8 \pi^{3} \emptyset \operatorname{Cos}[2 \pi(z-t)]\right)+ \\
L_{4}\left(4 \pi^{2} \emptyset \sin [2 \pi(z-t)]\right)+L_{5}(2 \pi \emptyset \cos [2 \pi(z-t)])+\left(M_{1}^{2}+\frac{1}{D_{a}}\right)-G r \mathcal{H}-G c C
\end{array}\right)-\frac{B_{2} r K_{1}\left(r b \sqrt{\left|s_{1}\right|}\right)}{b \sqrt{\left|s_{1}\right|}}- \\
& \frac{B_{4} r K_{1}\left(r b \sqrt{\left|s_{2}\right|}\right)}{b \sqrt{\left|s_{2}\right|}}+\frac{1}{2} B_{1} r^{2} \quad{ }_{0} \tilde{F}_{1}\left[2 ; \frac{r^{2}\left(b \sqrt{\left|s_{1}\right|}\right)^{2}}{4}\right]+\frac{1}{2} B_{3} r^{2} \quad{ }_{0} \tilde{F}_{1}\left[2 ; \frac{r^{2}\left(b \sqrt{\left|s_{2}\right|}\right)^{2}}{4}\right]
\end{aligned}
$$

where $K_{1}$ "is the modified Bessel function" of the second kind and $\left({ }_{0} \widetilde{F}_{1}\right)$ regular hypergeometricfunction.

\section{Discussion and The Numerical Results}

The numerical and computational results for the problem of an incompressible nonNewtonian Jeffrey fluid via porous media with heat and concentration with an inclination in the magnetic field are addressed with illustrations in this part. Figures 2-48 illustrate the key features of peristaltic flow of a couple-stress fluid through a porous material. The "MATHEMATICA 12" application was used for numerical findings and graphics.

\subsection{Temperature Distribution}

Basis on equation (37), figures 2-7 illustrates an effect the parameters $\Omega, \varepsilon, \emptyset, R n, t$ and $\operatorname{Pr}$ on the function of a fluid temperature $\mathcal{H}$ vs. $r$. Figures $2-6$, shows that a temperature of the fluid increases with increasing the parameters $\Omega, \varepsilon, R n, t$ and $\operatorname{Pr}$, respectively, while Figure 7 demonstrates that the temperature of a fluid decreases with increasing the parameter $\emptyset$.

\subsection{Concentration Distribution}

On the basis of equation (39), figures 8-15, illustrates parameter effects $\Omega, \epsilon, \emptyset, S r, S c, R n$, $\operatorname{Pr}$ and $t$ on the concentration functionC. Figure8, we notice that the concentration of fluid increases with increasing of $\Omega$. Infigure 9 , we notice the effect of the parameter $\epsilon$ on the concentration increases with increasing of $\epsilon$. By figure 10, We found the concentration decreases with increasing $\emptyset$. Figure 11, concentration change increases with increasing $S r$. Figure 12, we notice that $C$ increases withincreasing $S c$. Figure 13, the concentration increases with increasing $R n$. Figure 14, concentration change increases with increasing $P r$. Figure 15, the concentration increases with increasing $t$.

\subsection{Velocity Distribution}

On the basis of equation (40), figures 16-35 illustrates parameter results $\varepsilon, \emptyset, \lambda_{1}, L_{5}, L_{4}, L_{3}$, $L_{1}, \mathrm{t}, L_{2}, D a, \alpha, \zeta, M, G r, \Omega, P r, R n, S r, S c$ and $G c$ on the distribution of velocity $w$ vs.r. Figure 16, demonstrates the effect of the parameters $\varepsilon$ on the velocity distribution $w$ vs. $r$. It's found the velocity $w$ increases with increasing $\varepsilon$ at $r>0.12$, when there's $w$ decrease swith increase of $\varepsilon$ at $r<0.12$. Figure 17, shows the behavior of $w$ under the variation of $\emptyset$, we noticethe increase in the velocity when increasing $\emptyset$ in the region $r<0.2$ and the decrease in the velocity with increasing $\emptyset$ in the region $r>0.2$. Figure 18, we notice the rotation of the 
effect of the parameter $\lambda_{1}$, it is increase in the velocitywhen increasing $\lambda_{1}$ in theregion $r>0.2$ and $w$ decrease in the velocity with increasing $\lambda_{1}$ in the region $r<0.2$. Figure 19, we notice the rotation of the effect of the parameter $L_{5}$, it is increase in the velocitywhen increasing $L_{5}$ in the region $r<0.2$ and $w$ decrease in the velocity with increasing $L_{5}$ in the region $r>0.2$. Figure 20, we notice the rotation of the effect of the parameter $L_{4}$, it is increasein the velocity when increasing $L_{4}$ in the region $r<0.2$ and $w$ decrease in the velocity with increasing $L_{4}$ in the region $r>0.2$. Figure 21, shows the behavior of $w$ under the variation of $L_{3}$, we notice the increase in the velocity when increasing $L_{3}$ in the region $r>0.2$ and the decrease in the velocity with increasing $L_{3}$ in the region $r<0.2$. Figure 22, shows the behavior of $w$ under the variation of $L_{1}$, we notice the increase in the velocity when increasing $L_{1}$ in the region $r<0.2$ and the decrease in the velocity with increasing $L_{1}$ in the region $r>0.2$. Figure 23, shows the behavior of $w$ under the variation of $t$, we notice the increase in the velocity when increasing $t$ in the region $r>0.2$ and the decrease in the velocity with increasing $t$ in the region $r<0.2$. Figure 24, shows the behavior of $w$ under the variation of $L_{2}$, we notice the increase in the velocity when increasing $L_{2}$ in the region $r<0.2$ and the decrease in the velocity with increasing $L_{2}$ in the region $r>0.2$. In the figure 25, shows the behaviorof $w$ under the variation of $D a$, we notice the increase in the velocity when increasing $D a$ in the region $r<0.2$ and the decreasein the velocity with increasing $D a$ in the region $r>0.2$. In the figure 26, shows the behavior of $w$ under the variation of $\alpha$, we notice the increase in the velocity when increasing $\alpha$ in the region $r<0.2$ and the decrease in the velocity with increasing $\alpha$ in the region $r>0.2$. In the figure 27, shows the behavior of $w$ under the variation of $\zeta$, we notice the decrease in the velocity when increasing $\zeta$. Figure 28, shows the behavior of $w$ under the variation of $M$, we notice the increase in the velocity when increasing $M$ in the region $r>0.2$ and the decreasein the velocity with increasing $M$ in the region $r<0.2$. Figure 29, shows the behavior of $w$ under the variation of $G r$, we notice the decrease in the velocity when increasing $G r$ in the region $r<0.2$ and the increasein the velocity with increasing $G r$ in the region $r>0.2$.

Figure 30, shows the behavior of $w$ under the variation of $\Omega$, we notice the decrease in the velocity when increasing $\Omega$. In the figure 31, shows the behavior of $w$ under the variation of $P r$, we notice the decrease in the velocity when increasing $P r$. We notice the effect of $R n$, on the velocity functionin figure 32, shows the behavior of $w$ is similar to the effect of $M$ in figure 28. As figures 33 and 34 shows an decreases in velocitywith the increase of both parameters $S r$ and $S c$. We notice the effectof $G c$ on the velocity functionin figure 35, $w$ is similar to the effect of $M$ in figure 28.

\subsection{Trapping Phenomena}

The development of an internally circulating bolus of fluid by closed streamlines is known as trapping, and this trapped bolus is pushed forward with the peristaltic wave. According to equation (42), the effects $\varepsilon, \emptyset, \lambda_{1}, L_{5}, L_{4}, L_{3}, L_{1}, \mathrm{t}, L_{2}, D a, \alpha, \zeta, M, G r, \Omega, P r, R n, S r, S c$ and Gc ontrapping can be seen through 36 - 48. Figure 36, shows that the volume of the trapped boluslowered with the increase $\varepsilon$ graduallyin the middleof the channel. In the figure 37 , shows that the sizeof the trapped bolus located in the center of the channel increases with the increase $\varnothing$. By figure 38 , the volume of the trapped bolus decreases when increase $\lambda_{1}$ gradually in middle of channel. By figure 39, we notice trapped bolus, in the centerof the channel increase with increase $L_{5}$ the bolus will turn into a wave and $L_{5}$ has a weak impact. In figure 40, with the increases $L_{4}$ gradually the size of the trapped bolus increasesat the middle. In figure 41 , the volumeof the trapped bolus its beginning to grow in the center with increaseof $L_{3}$. Figure 42 , showseffect the parameter $L_{1}$ on trappedbolus, as that caused the bolusto grow in the centerof the channelwith increase of $L_{1}$. In figure 43 , the volumeof the trapped bolusin the middle decreases with $t$ increaseand the wave will turn into a bolus. In figure 44, the volumeof the trappedbolus in the waveat the center increases with $L_{2}$ increase and we get wavesof bolus. In figure 45 , the volumeof the trapped bolus in the waveincreases with $D a$ increaseand the bolus willturn into awave. In figure 46, with $\alpha$ increasying we getincrease the volumof the 
trapped bolusin the waveat the center. By figure 47, with $\zeta$ increasying weget increase the volumeof the trappedbolus in the waveat the center. Figure 48, the volumeof the trapped bolusin the middle decreases with $M$ increaseand the wave will turnin to a bolus. And finally, we note that the following parameters are $G r, \Omega, P r, R n, S r, S c$ and $G c$ very weak.

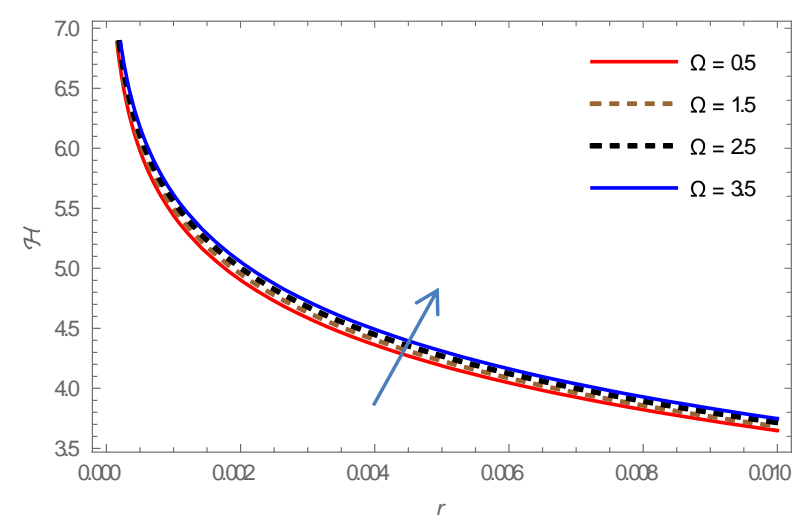

Figure 2: Temperature variations $\mathcal{H}$ vs. $r$ at $\epsilon=0.3, \emptyset=0.3, \operatorname{Rn}=0.1, \operatorname{Pr}=2, \quad t=0.05$, $z=0.1$.

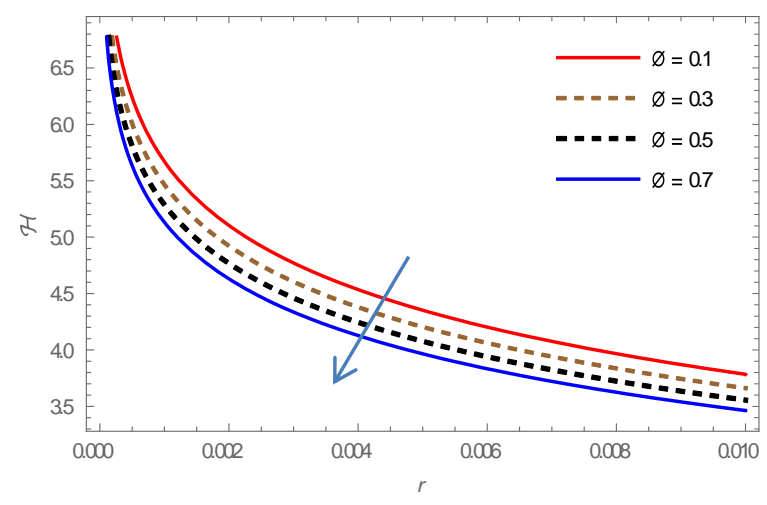

Figure 4: Temperature variations $\mathcal{H}$ vs. $r$ at $\Omega=0.9, \epsilon=0.3, R n=0.1, \operatorname{Pr}=2, t=0.05$, $z=0.1$.

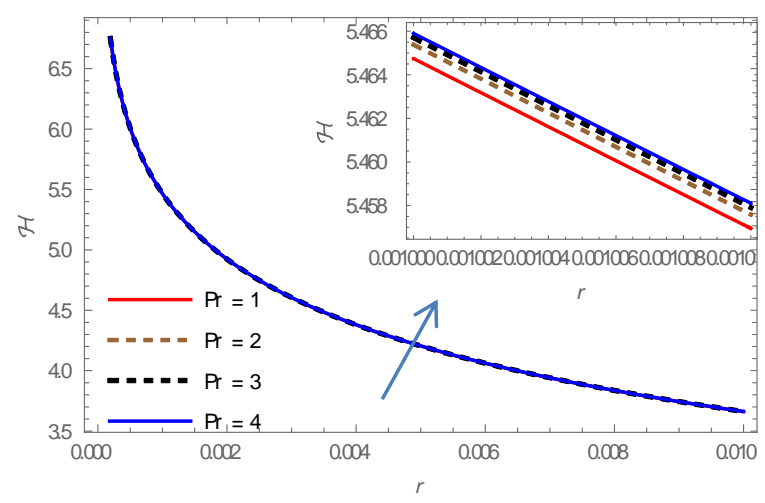

Figure 6: Temperature variations $\mathcal{H}$ vs. $r$ at $\Omega=0.9, \epsilon=0.3, R n=0.1, \emptyset=0.3, t=0.05$, $z=0.1$.

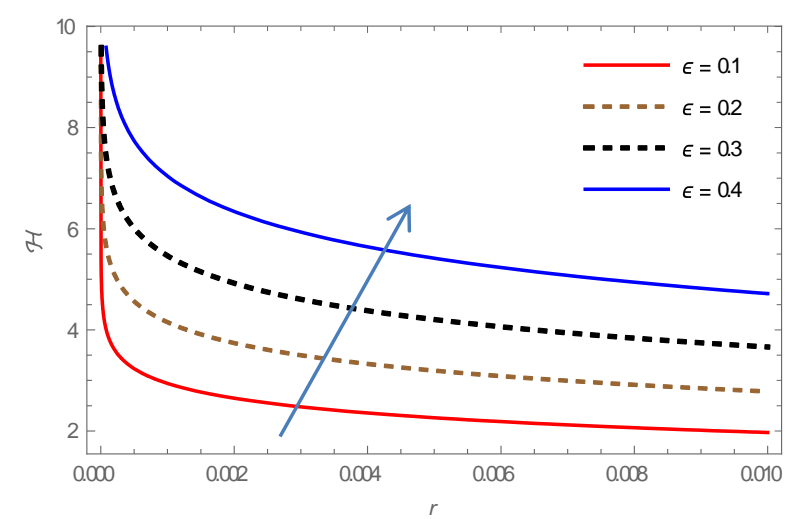

Figure 3: Temperature variations $\mathcal{H}$ vs. $r$ at $\Omega=0.9, \varnothing=0.3, R n=0.1, \operatorname{Pr}=2, t=0.05$, $z=0.1$.

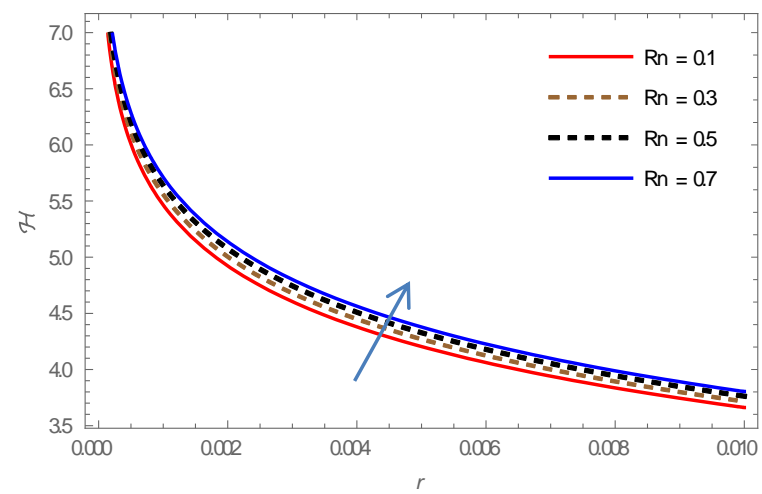

Figure 5: Temperature variations $\mathcal{H}$ vs. $r$ at $\Omega=0.9, \varnothing=0.3, \epsilon=0.3, \operatorname{Pr}=2, t=0.05$, $z=0.1$.

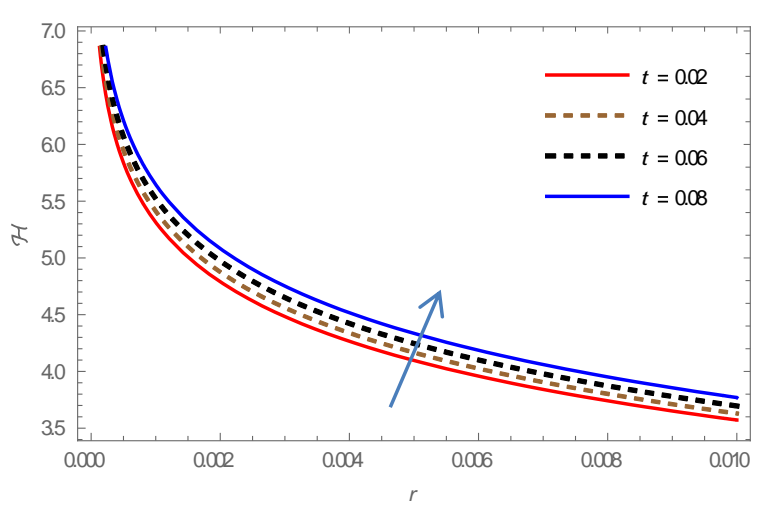

Figure 7: Temperature variations $\mathcal{H}$ vs. $r$ at $\Omega=0.9, \quad \epsilon=0.3, \quad R n=0.1, \quad \operatorname{Pr}=2, \quad \varnothing=0.3$, $z=0.1$. 


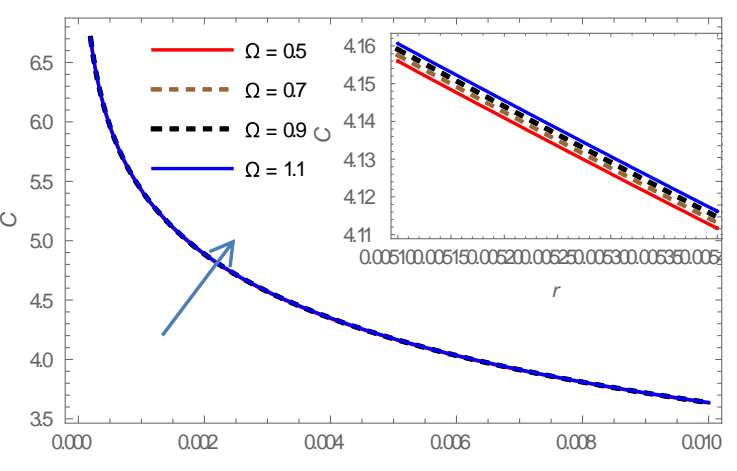

Figure 8: concentration variation $C$ vs. $r$ at $\varepsilon=0.2, \emptyset=0.2, S r=0.6, S c=0.5, \quad R n=$ $0.5, \operatorname{Pr}=2, t=0.05, z=0.1$.

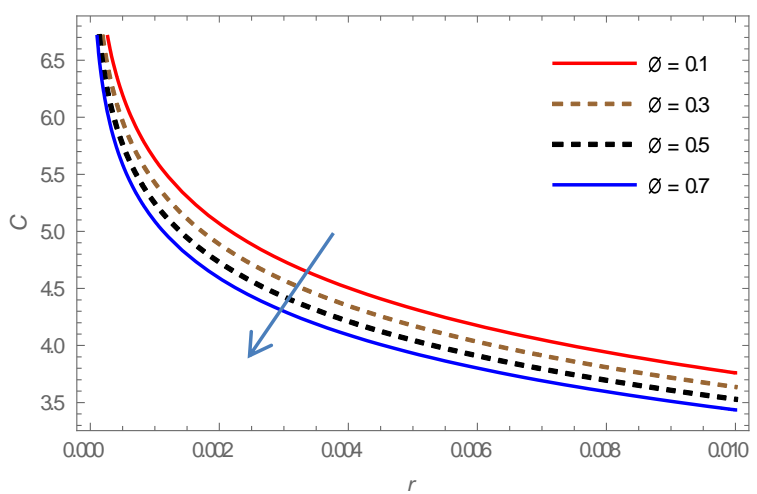

Figure 10: concentration variation $C$ vs. $r$ at $\Omega=0.2, \varepsilon=0.2, S r=0.6, S c=0.5, R n=0.5$, $\operatorname{Pr}=2, t=0.05, z=0.1$.

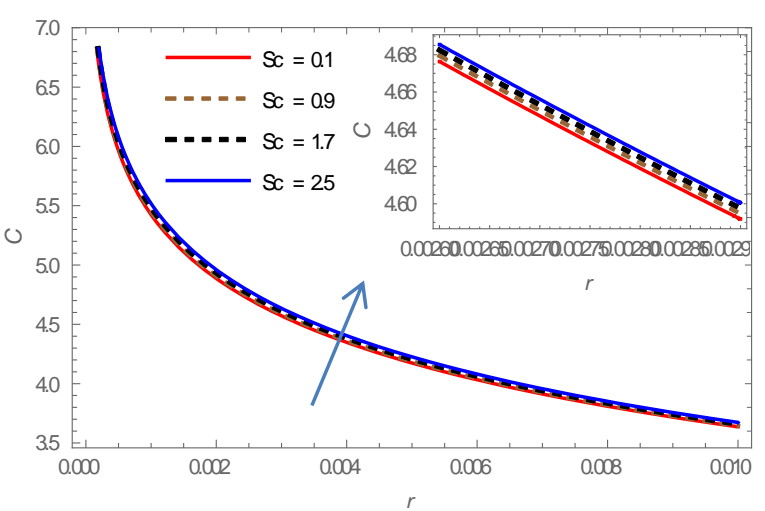

Figure 12: concentration variation $C$ vs. $r$ at $\Omega=$ $0.2, \varepsilon=0.2, \emptyset=0.2, S r=0.6, R n=0.5, \operatorname{Pr}=2$, $t=0.05, z=0.1$.

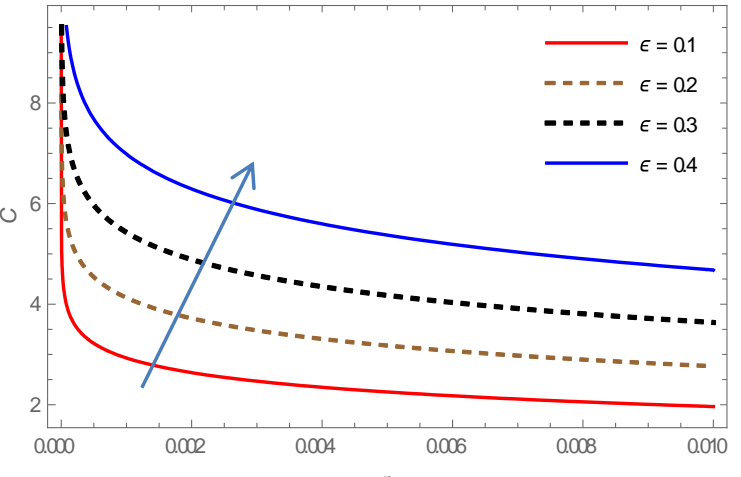

Figure 9: concentration variation $C$ vs. $r$ at $\Omega=0.2, \emptyset=0.2, S r=0.6, S c=0.5, R n=0.5$, $\operatorname{Pr}=2, t=0.05, z=0.1$.

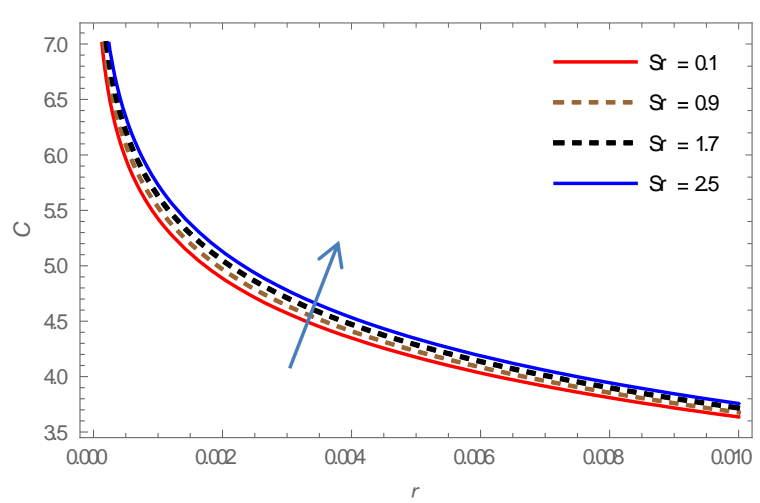

Figure 11: concentration variation $C$ vs. $r$ at $\Omega=0.2, \varepsilon=0.2, \emptyset=0.2, S c=0.5, R n=0.5$, $\operatorname{Pr}=2, t=0.05, z=0.1$.

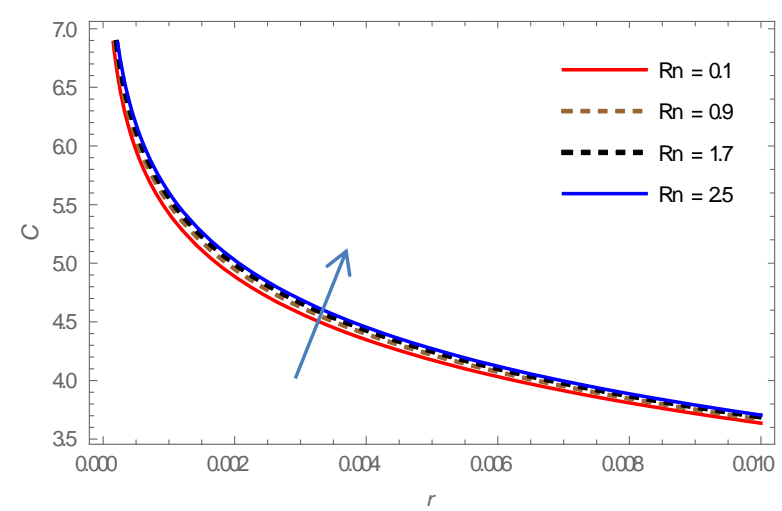

Figure 13: concentration variation $\mathrm{C}$ vs. $r$ at $\Omega=0.2, \varepsilon=0.2, \emptyset=0.2, S r=0.6, S c=0.5$, $P r=2, t=0.05, z=0.1$. 


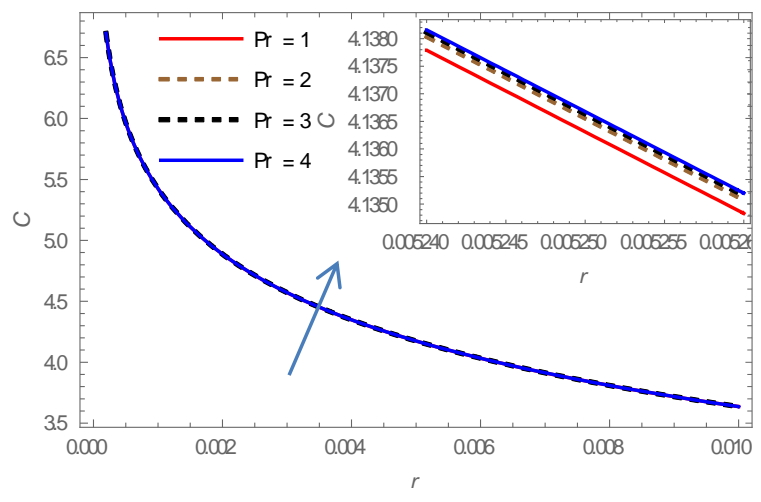

Figure 14: concentration variation $\mathrm{C}$ vs. $r$ at $\Omega=0.2, \varepsilon=0.2, \emptyset=0.2, S r=0.6, S c=0.5$, $R n=0.5, t=0.05, z=0.1$.

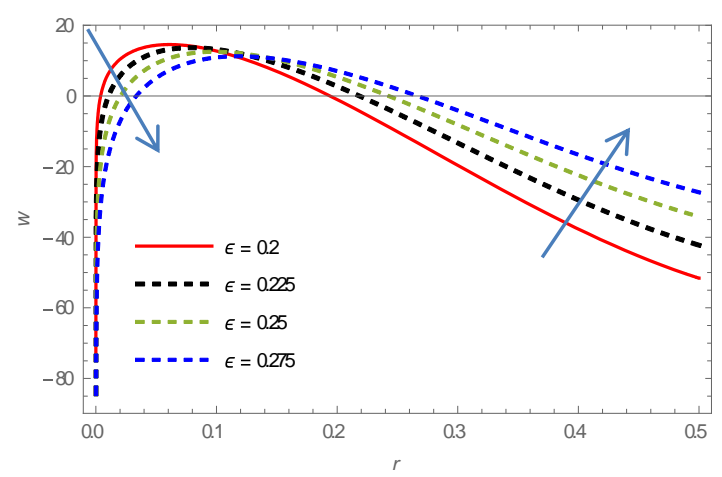

Figure 16: velocity distribution for various values of $\varepsilon$ with $\varnothing=0.2, \lambda_{1}=0.1, L_{5}=0.1, L_{4}=0.5$, $L_{3}=0.1, L_{1}=0.1, t=0.05, L_{2}=0.5, D a=0.9$, $\alpha=3.75, \quad \zeta=0.5, \quad M=1.1, \quad G r=2, \Omega=0.9$, $\operatorname{Pr}=2, R n=0.5, \quad S r=0.6, S c=0.5, G c=1$, $z=0.1$.

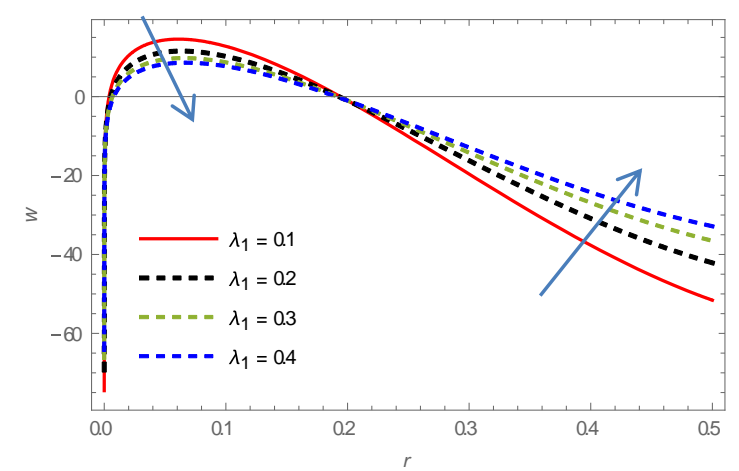

Figure 18: velocity distribution for various values of $\lambda_{1}$ with $\varepsilon=0.2, \varnothing=0.2, L_{5}=0.1, L_{4}=0.5$, $L_{3}=0.1, L_{1}=0.1, t=0.05, L_{2}=0.5, D a=0.9$, $\alpha=3.75, \quad \zeta=0.5, \quad M=1.1, \quad G r=2, \quad \Omega=0.9$, $\operatorname{Pr}=2, \quad R n=0.5, \quad S r=0.6, S c=0.5, \quad G c=1$, $z=0.1$.

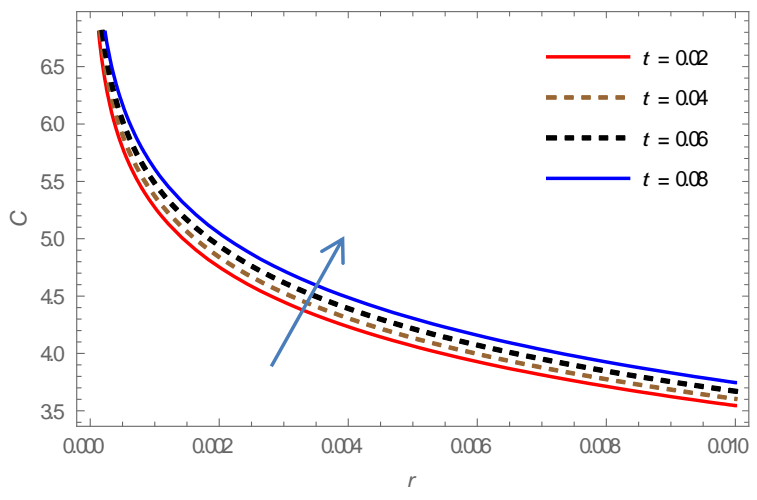

Figure 15: concentration variation $C$ vs. $r$ at $\Omega=0.2, \varepsilon=0.2, \emptyset=0.2, S r=0.6, S c=0.5$, $R n=0.5, \operatorname{Pr}=2, z=0.1$.

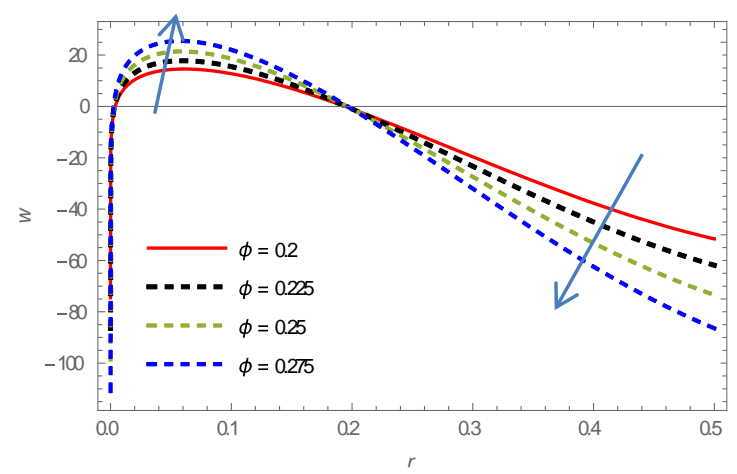

Figure 17: velocity distribution for various values of $\emptyset$ with $\varepsilon=0.2, \lambda_{1}=0.1, L_{5}=0.1, L_{4}=0.5$, $L_{3}=0.1, L_{1}=0.1, t=0.05, L_{2}=0.5, D a=0.9$, $\alpha=3.75, \quad \zeta=0.5, \quad M=1.1, \quad G r=2, \Omega=0.9$, $\operatorname{Pr}=2, R n=0.5, S r=0.6, S c=0.5, G c=1$, $z=0.1$.

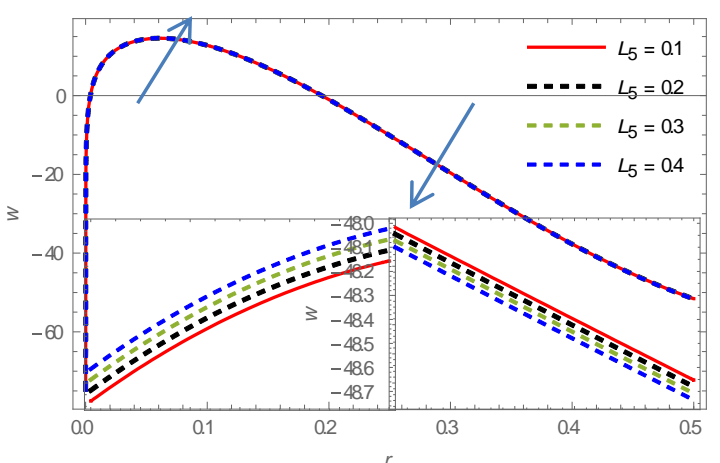

Figure 19: velocity distribution for various values of $L_{5}$ with $\varepsilon=0.2, \emptyset=0.2, \lambda_{1}=0.1, L_{4}=0.5$, $L_{3}=0.1, L_{1}=0.1, t=0.05, L_{2}=0.5, D a=0.9$, $\alpha=3.75, \quad \zeta=0.5, \quad M=1.1, \quad G r=2, \quad \Omega=0.9$, $\operatorname{Pr}=2, R n=0.5, \quad S r=0.6, S c=0.5, G c=1$, $z=0.1$. 


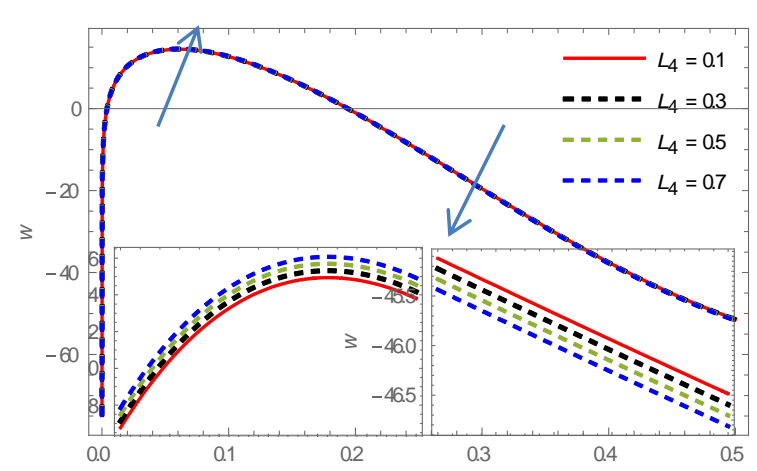

Figure 20: velocity distribution for various values of $L_{4} \quad$ with $\varepsilon=0.2, \quad \emptyset=0.2, \quad \lambda_{1}=0.1, \quad L_{5}=0.1$ $L_{3}=0.1, L_{1}=0.1, t=0.05, L_{2}=0.5, \quad D a=0.9$, $\alpha=3.75, \zeta=0.5, M=1.1, G r=2, \Omega=0.9, \operatorname{Pr}=$ 2, $R n=0.5, S r=0.6, S c=0.5, G c=1, z=0.1$.

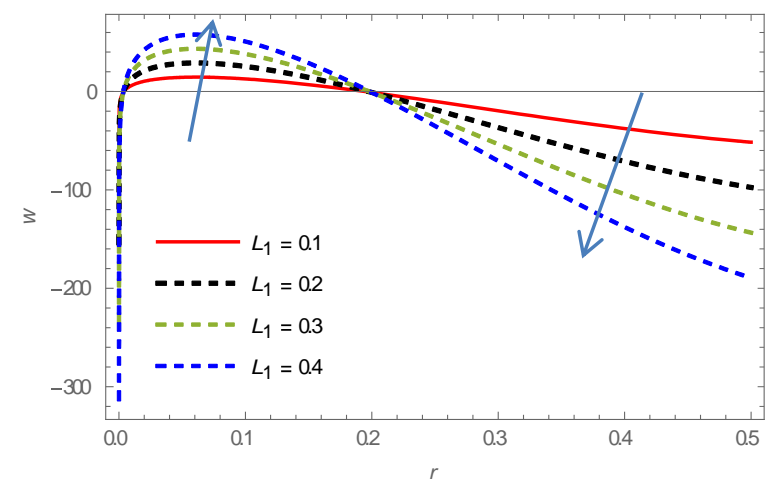

Figure 22: velocity distribution for various values of $L_{1}$ with $\varepsilon=0.2, \varnothing=0.2, \lambda_{1}=0.1, L_{5}=0.1, L_{4}=$ $0.5, L_{3}=0.1, \quad t=0.05, L_{2}=0.5, \quad D a=0.9, \quad \alpha=$ $3.75, \zeta=0.5, M=1.1, G r=2, \Omega=0.9, P r=2$, $R n=0.5, S r=0.6, S c=0.5, G c=1, z=0.1$.

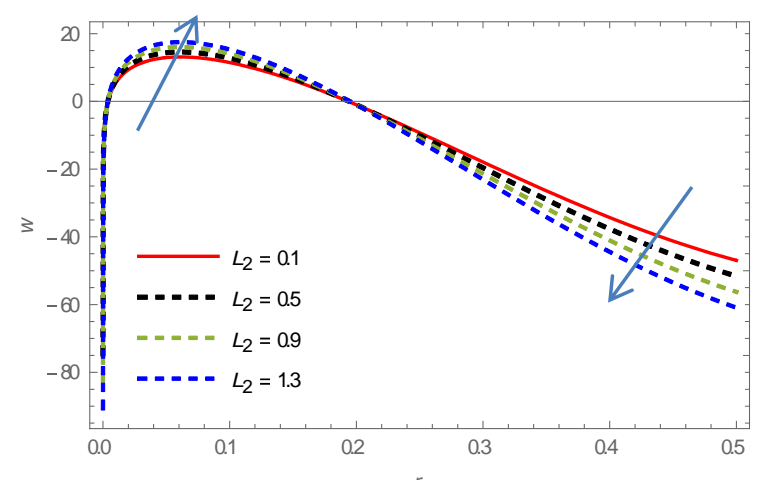

Figure 24: velocity distribution for various values of $L_{2}$ with $\varepsilon=0.2, \emptyset=0.2, \lambda_{1}=0.1, L_{5}=0.1, L_{4}=0.5$, $L_{3}=0.1, \quad L_{1}=0.1, \quad \mathrm{t}=0.05, \quad D a=0.9, \quad \alpha=3.75$, $\zeta=0.5, \quad M=1.1, \quad G r=2, \Omega=0.9, \quad P r=2, \quad R n=$ $0.5, S r=0.6, S c=0.5, G c=1, z=0.1$.

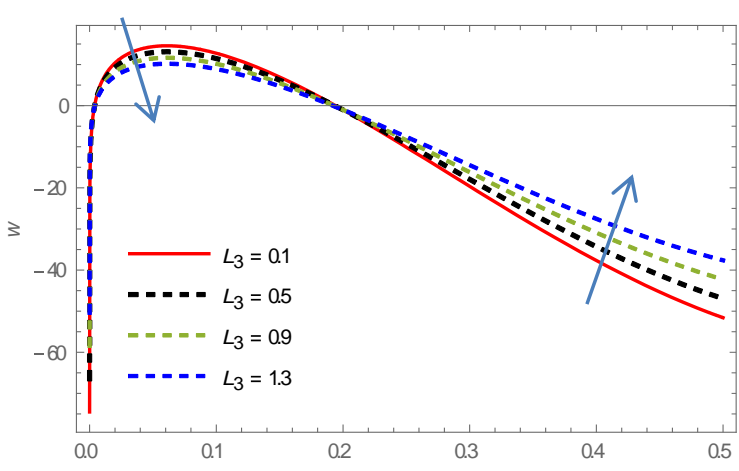

Figure 21: velocity distribution for various values of $L_{3} \quad$ with $\varepsilon=0.2, \quad \varnothing=0.2, \quad \lambda_{1}=0.1, \quad L_{5}=0.1$, $L_{4}=0.5, L_{1}=0.1, t=0.05, L_{2}=0.5, \quad D a=0.9$, $\alpha=3.75, \zeta=0.5, M=1.1, G r=2, \Omega=0.9, P r=$ 2, $R n=0.5, S r=0.6, S c=0.5, G c=1, z=0.1$.

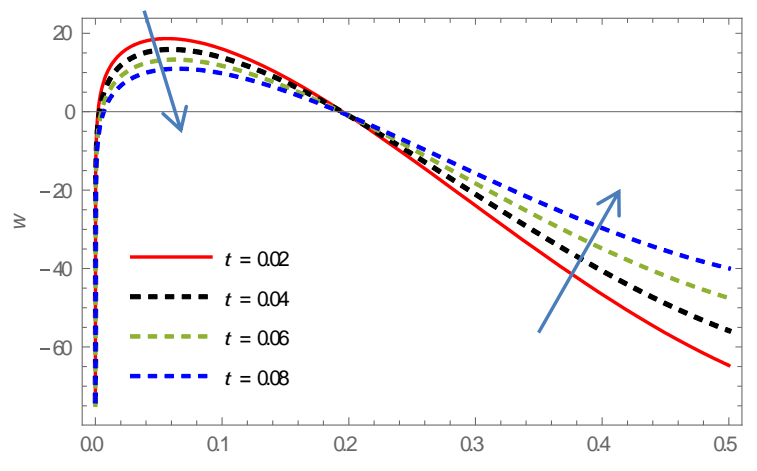

Figure 23: velocity distribution for various values of $t$ with $\varepsilon=0.2, \emptyset=0.2, \lambda_{1}=0.1, L_{5}=0.1, L_{4}=0.5$, $L_{3}=0.1, L_{1}=0.1, L_{2}=0.5, D a=0.9, \alpha=3.75$, $\zeta=0.5, \quad M=1.1, \quad G r=2, \quad \Omega=0.9, \quad \operatorname{Pr}=2$, $R n=0.5, S r=0.6, S c=0.5, G c=1, z=0.1$.

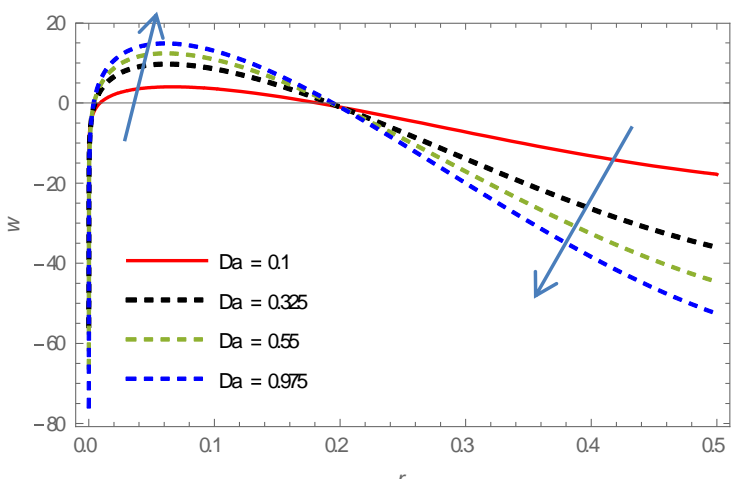

Figure 25: velocity distribution for various values of $D a$ with $\varepsilon=0.2, \varnothing=0.2, \lambda_{1}=0.1, L_{5}=0.1, L_{4}=0.5$, $L_{3}=0.1, \quad L_{1}=0.1, \quad \mathrm{t}=0.05, \quad L_{2}=0.5, \quad \alpha=3.75$, $\zeta=0.5, M=1.1, \quad G r=2, \Omega=0.9, \quad P r=2, \quad R n=$ $0.5, S r=0.6, S c=0.5, G c=1, z=0.1$. 


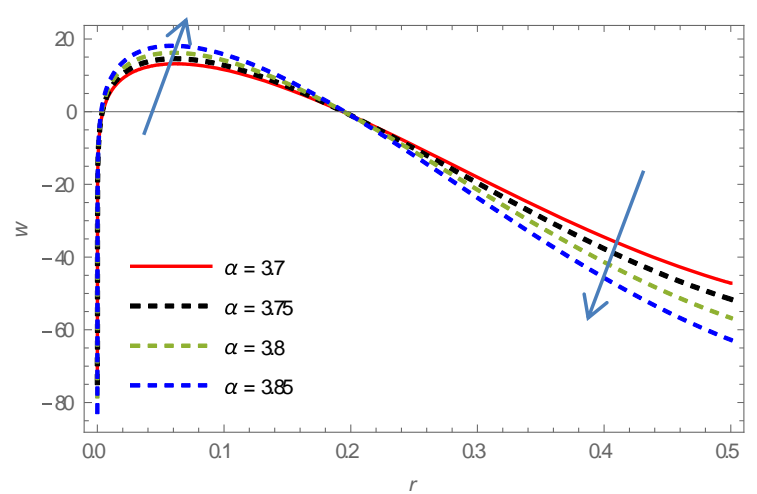

Figure 26: velocity distribution for various values of $\alpha$ with $\varepsilon=0.2, \varnothing=0.2, \lambda_{1}=0.1, L_{5}=0.1, L_{4}=0.5$, $L_{3}=0.1, L_{1}=0.1, \mathrm{t}=0.05, L_{2}=0.5, \quad D a=0.9$, $\zeta=0.5, M=1.1, G r=2, \Omega=0.9, \quad P r=2, R n=$ $0.5, S r=0.6, S c=0.5, G c=1, Z=0.1$.

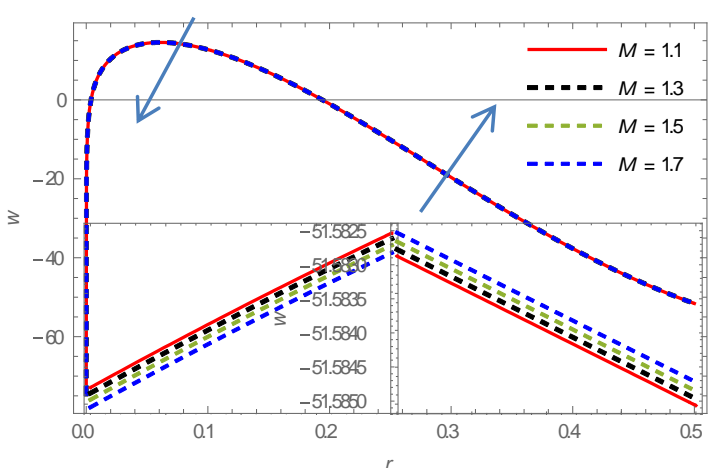

Figure 28: velocity distribution for various values of $M$ with $\varepsilon=0.2, \varnothing=0.2, \lambda_{1}=0.1, L_{5}=0.1, L_{4}=0.5$, $L_{3}=0.1, \quad L_{1}=0.1, \quad t=0.05, \quad L_{2}=0.5, \quad D a=0.9$, $\alpha=3.75, \zeta=0.5, \quad G r=2, \Omega=0.9, \operatorname{Pr}=2, R n=$ $0.5, S r=0.6, S c=0.5, G c=1, z=0.1$.

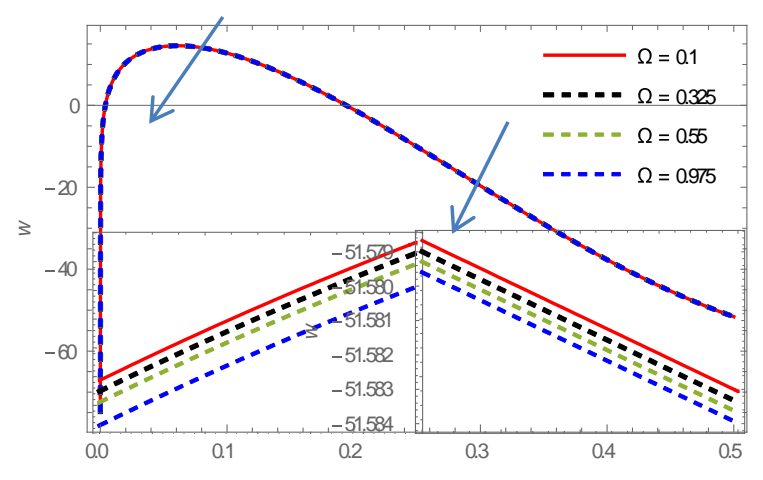

Figure 30: velocity distribution for various values of $\Omega$ with $\varepsilon=0.2, \emptyset=0.2, \lambda_{1}=0.1, L_{5}=0.1, L_{4}=0.5$, $L_{3}=0.1, \quad L_{1}=0.1, \quad t=0.05, \quad L_{2}=0.5, \quad D a=0.9$, $\alpha=3.75, \quad \zeta=0.5, \quad M=1.1, \quad G r=2, \quad \operatorname{Pr}=2$, $R n=0.5, S r=0.6, S c=0.5, G c=1, z=0.1$.

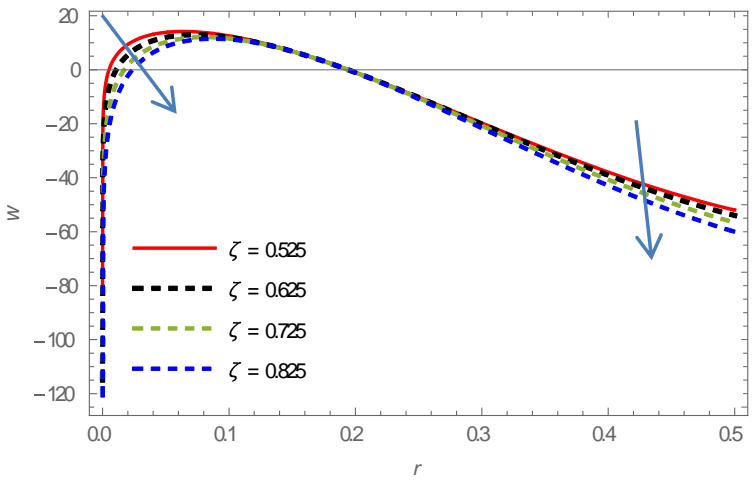

Figure 27: velocity distribution for various values of $\zeta$ with $\varepsilon=0.2, \emptyset=0.2, \lambda_{1}=0.1, L_{5}=0.1, L_{4}=0.5$, $L_{3}=0.1, \quad L_{1}=0.1, \mathrm{t}=0.05, \quad L_{2}=0.5, \quad D a=0.9$, $\alpha=3,75, \quad M=1.1, \quad G r=2, \quad \Omega=0.9, \quad \operatorname{Pr}=2$, $R n=0.5, S r=0.6, S c=0.5, G c=1, z=0.1$.

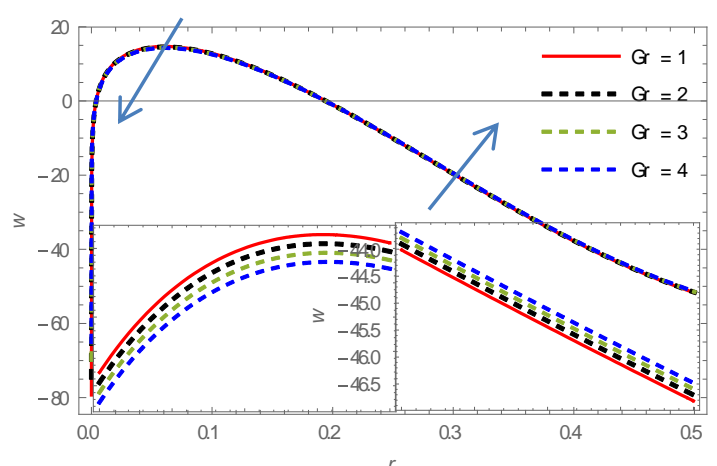

Figure 29: velocity distribution for various values of $G r$ with $\varepsilon=0.2, \varnothing=0.2, \lambda_{1}=0.1, L_{5}=0.1, L_{4}=0.5$, $L_{3}=0.1, \quad L_{1}=0.1, \quad t=0.05, \quad L_{2}=0.5, \quad D a=0.9$, $\alpha=3.75, \zeta=0.5, M=1.1, \Omega=0.9, \operatorname{Pr}=2, \quad R n=$ $0.5, S r=0.6, S c=0.5, G c=1, z=0.1$.

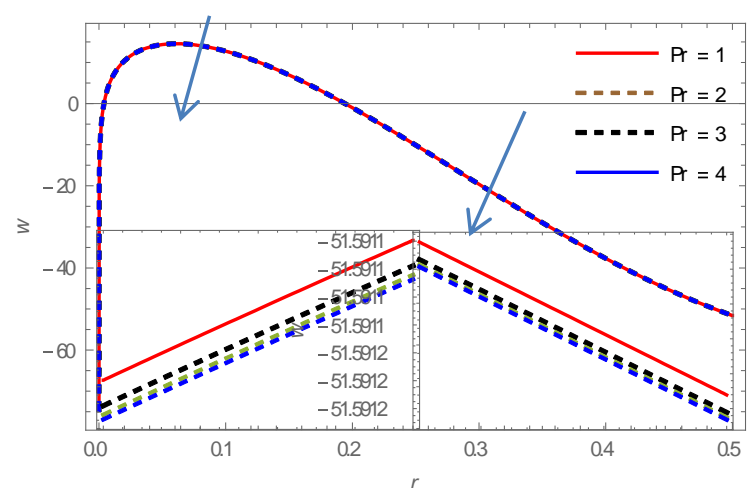

Figure 31: velocity distribution for various values of $\mathrm{Pr}$ with $\varepsilon=0.2, \emptyset=0.2, \lambda_{1}=0.1, L_{5}=0.1, L_{4}=0.5$, $L_{3}=0.1 \quad L_{1}=0.1, \quad t=0.05, \quad L_{2}=0.5, \quad D a=0.9$, $\alpha=3.75, \quad \zeta=0.5, \quad M=1.1, \quad G r=2, \quad \Omega=0.9$, $R n=0.5, S r=0.6, S c=0.5, G c=1, z=0.1$. 


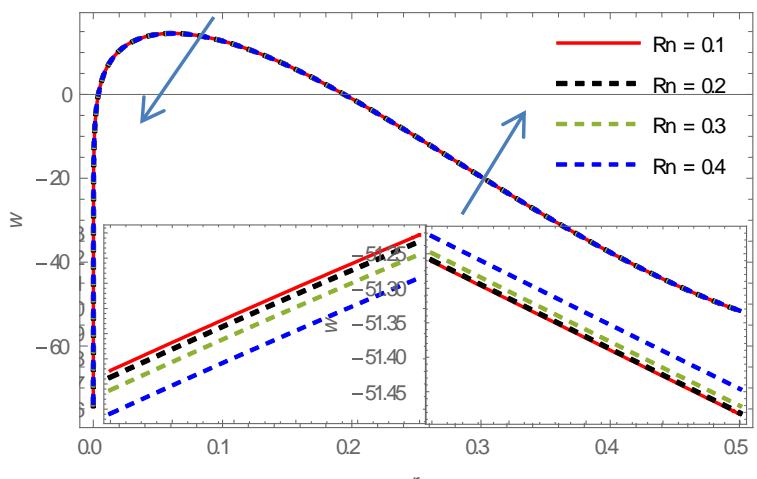

Figure 32: velocity distribution for various values of $R n$ with $\varepsilon=0.2, \varnothing=0.2, \lambda_{1}=0.1, L_{5}=0.1, L_{4}=$ $0.5, L_{3}=0.1, L_{1}=0.1, t=0.05, L_{2}=0.5, D a=0.9$, $\alpha=3.75, \quad \zeta=0.5, \quad M=1.1, \quad G r=2, \quad \Omega=0.9$, $\operatorname{Pr}=2, S r=0.6, S c=0.5, G c=1, z=0.1$.

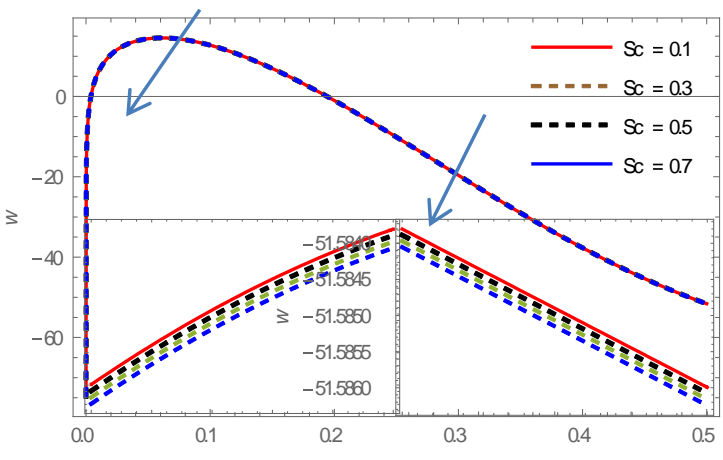

Figure 34: velocity distribution for various values of $S c$ with $\varepsilon=0.2, \emptyset=0.2, \lambda_{1}=0.1, L_{5}=0.1, L_{4}=0.5$, $L_{3}=0.1, \quad L_{1}=0.1, \quad t=0.05, \quad L_{2}=0.5, \quad D a=0.9$, $\alpha=3.75, \quad \zeta=0.5, \quad M=1.1, \quad G r=2, \quad \Omega=0.9$, $P r=2, R n=0.5, S r=0.6, G c=1, z=0.1$.

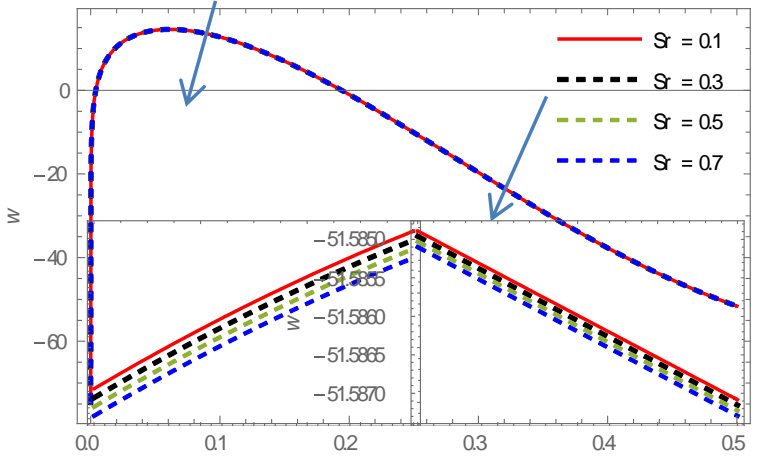

Figure 33: velocity distribution for various values of $\mathrm{Sr}$ with $\varepsilon=0.2, \emptyset=0.2, \lambda_{1}=0.1, L_{5}=0.1, L_{4}=0.5$, $L_{3}=0.1 \quad L_{1}=0.1, \quad t=0.05, \quad L_{2}=0.5, \quad D a=0.9$, $\alpha=3.75, \quad \zeta=0.5, \quad M=1.1, \quad G r=2, \quad \Omega=0.9$, $P r=2, R n=0.5, S c=0.5, G c=1, z=0.1$.

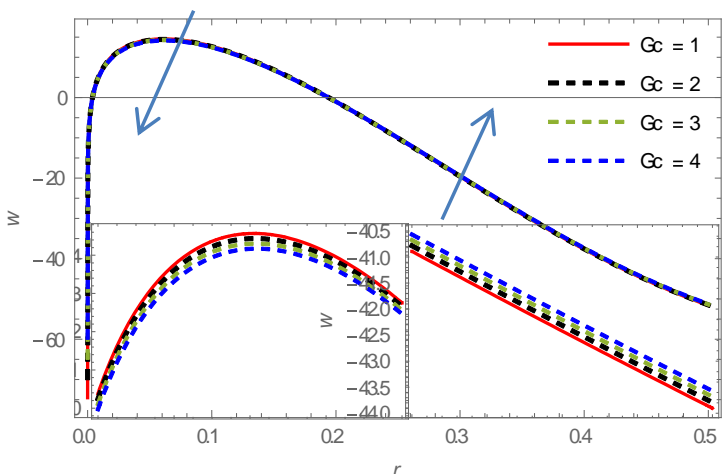

Figure 35: velocity distribution for various values of Gc with $\varepsilon=0.2, \varnothing=0.2, \lambda_{1}=0.1, L_{5}=0.1, L_{4}=$ $0.5, L_{3}=0.1 L_{1}=0.1, t=0.05, L_{2}=0.5, D a=0.9$, $\alpha=3.75, \quad \zeta=0.5, \quad M=1.1, \quad G r=2, \quad \Omega=0.9$, $\operatorname{Pr}=2, R n=0.5, S r=0.6, S c=0.5, z=0.1$.
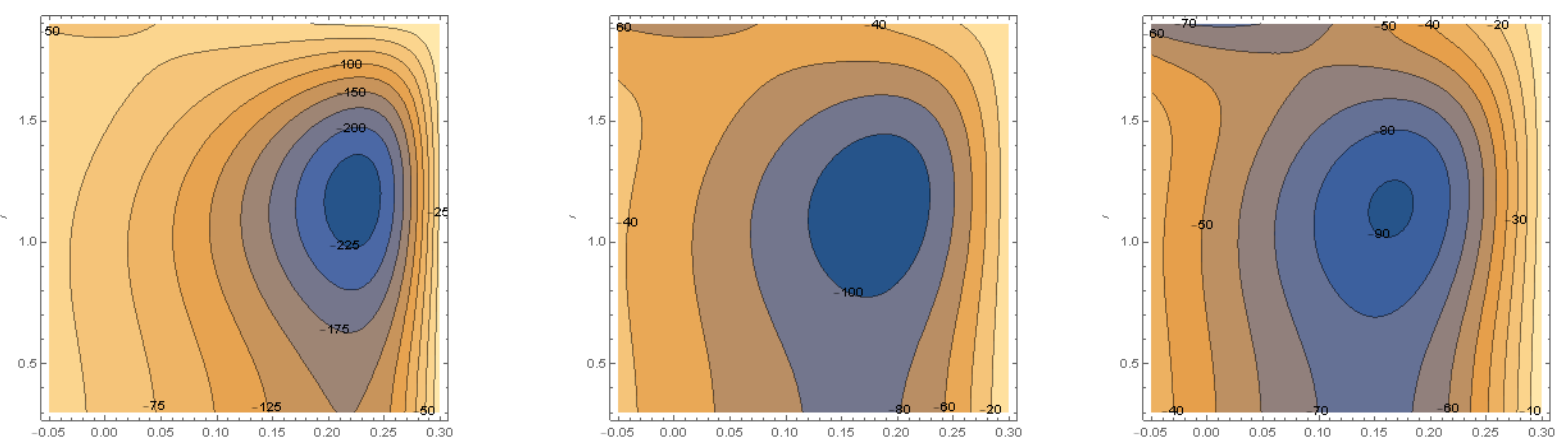

Figure 36: Wave frame streamlines for different values of $\varepsilon=\{0.1,0.2,0.3\}$ at $\phi=0.2, \lambda_{1}=0.1$, $L_{5}=0.1, L_{4}=0.5, L_{3}=0.1, L_{1}=0.1, t=0.05, L_{2}=0.5, \mathrm{Da}=0.9, \alpha=3.75, \zeta=0.5, M=$ 1.1, $G r=2, \Omega=0.9, P r=2, R n=0.5, S r=0.6, S c=0.5, G c=1$. 

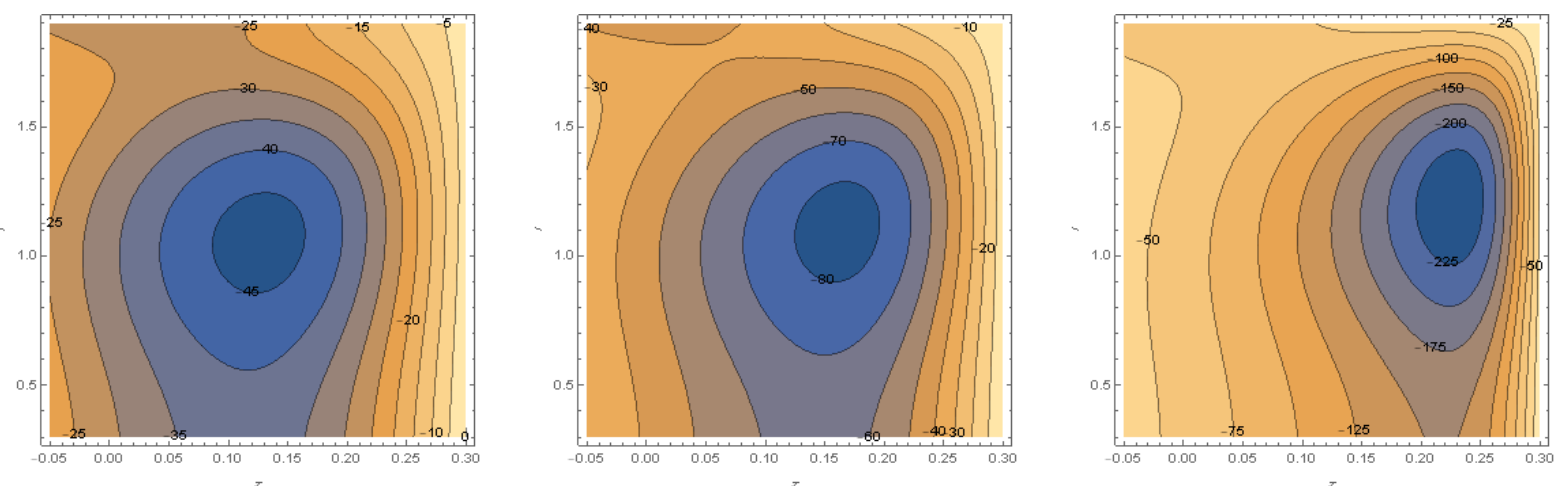

Figure 37: Wave frame streamlines for different values of $\phi=\{0.1,0.15,0.225\}$ at $\varepsilon=0.2, \lambda_{1}=0.1$, $L_{5}=0.1, L_{4}=0.5, \quad L_{3}=0.1, L_{1}=0.1, t=0.05, L_{2}=0.5, \mathrm{Da}=0.9, \alpha=3.75, \zeta=0.5, M=1.1, G r=$ $2, \Omega=0.9, \operatorname{Pr}=2, R n=0.5, S r=0.6, S c=0.5, G c=1$.
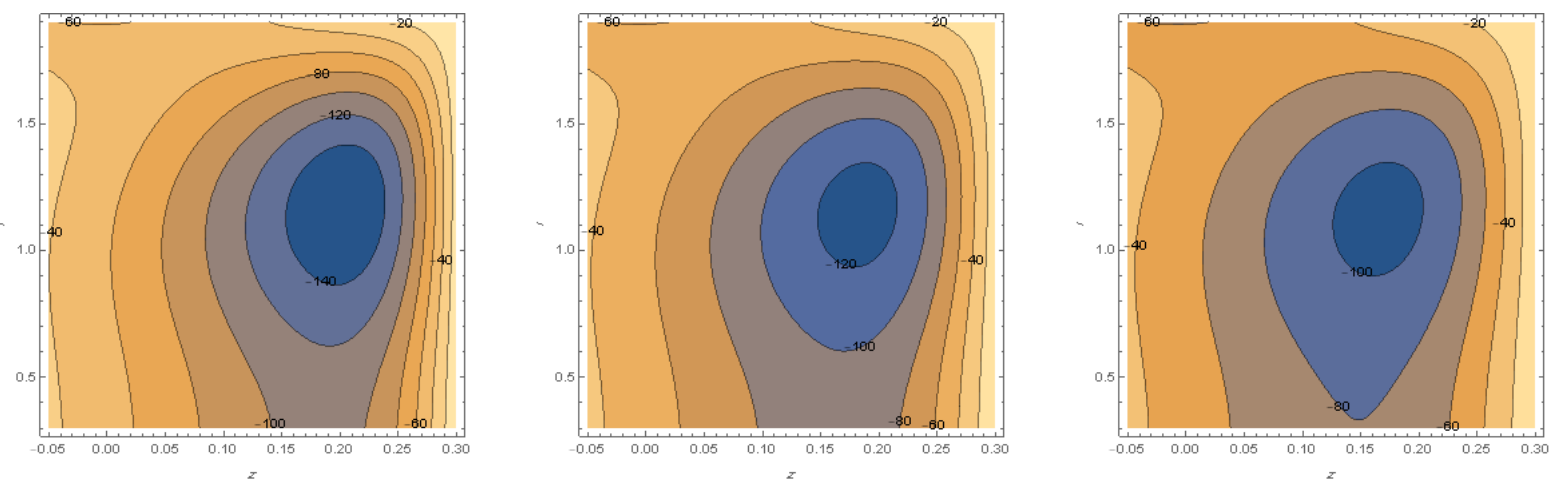

Figure 38: Wave frame streamlines for different values of $\lambda_{1}=\{0.1,0.15,0.225\}$ at $\varepsilon=0.2, \phi=0.2$, $L_{5}=0.1, L_{4}=0.5, \quad L_{3}=0.1, L_{1}=0.1, t=0.05, L_{2}=0.5, \mathrm{Da}=0.9, \alpha=3.75, \zeta=0.5, M=1.1, G r=$ $2, \Omega=0.9, \operatorname{Pr}=2, R n=0.5, S r=0.6, S c=0.5, G c=1$.
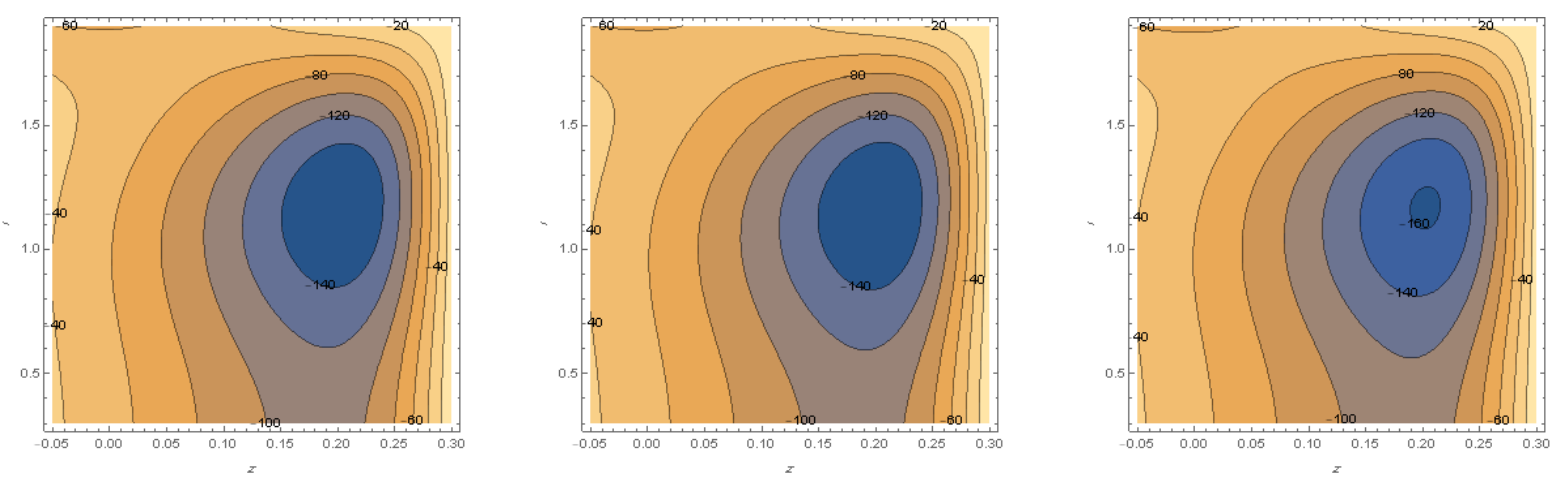

Figure 39: Wave frame streamlines for different values of $L_{5}=\{2,4,6\}$ at $\varepsilon=0.2, \phi=0.2, \lambda_{1}=0.1$, $L_{4}=0.5, \quad L_{3}=0.1, L_{1}=0.1, t=0.05, L_{2}=0.5, \mathrm{Da}=0.9, \alpha=3.75, \zeta=0.5, M=1.1, G r=2, \Omega=0.9$, $P r=2, R n=0.5, S r=0.6, S c=0.5, G c=1$. 

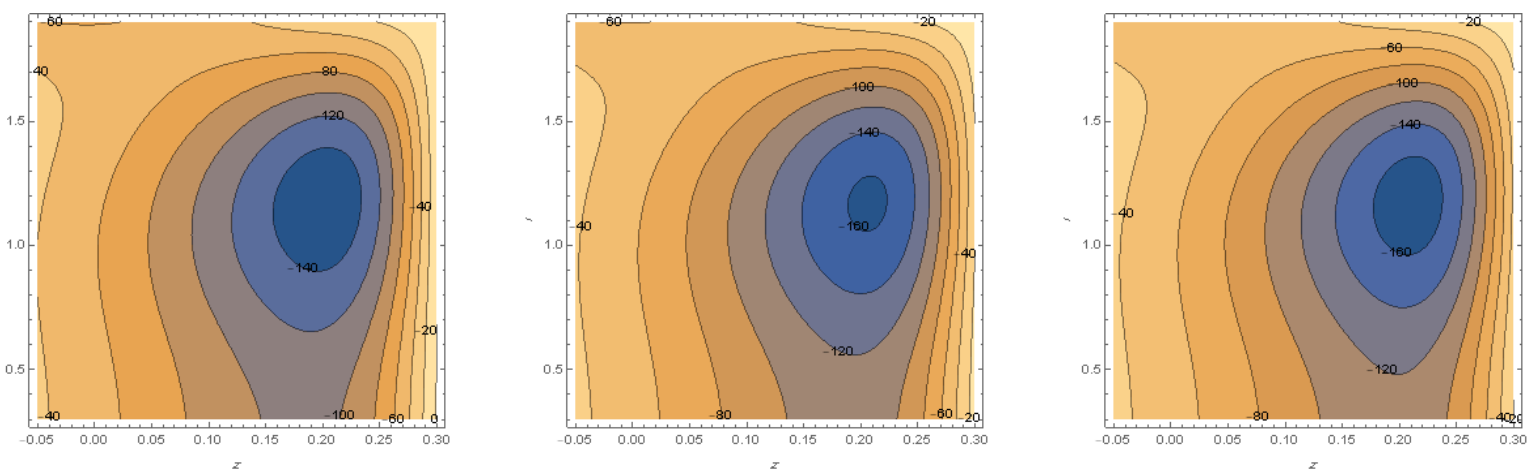

Figure 40: Wave frame streamlines for different values of $L_{4}=\{0.1,1.3,2.1\}$ at $\varepsilon=0.2, \phi=0.2, \lambda_{1}=0.1$, $L_{5}=0.1, \quad L_{3}=0.1, L_{1}=0.1, t=0.05, L_{2}=0.5, \mathrm{Da}=0.9, \alpha=3.75, \zeta=0.5, M=1.1, G r=2, \Omega=0.9$, $\operatorname{Pr}=2, R n=0.5, S r=0.6, S c=0.5, G c=1$.
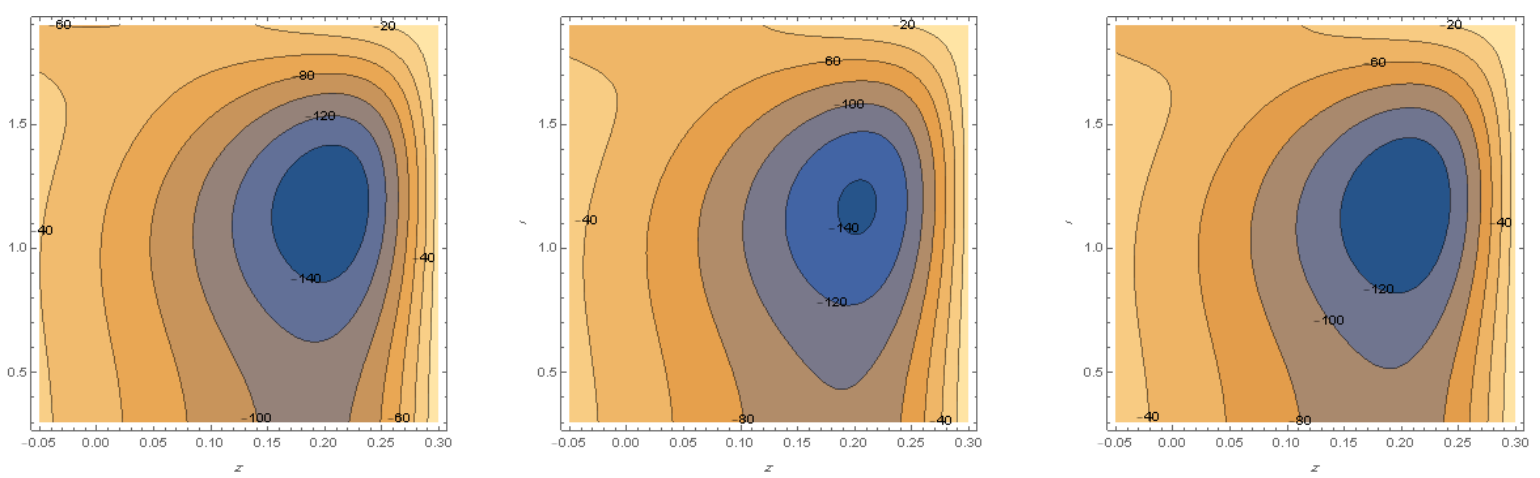

Figure 41: Wave frame streamlines for different values of $L_{3}=\{0.1,0.5,0.625\}$ at $\varepsilon=0.2, \phi=0.2, \lambda_{1}=0.1$, $L_{5}=0.1, L_{4}=0.5, L_{1}=0.1, t=0.05, L_{2}=0.5, \mathrm{Da}=0.9, \alpha=3.75, \zeta=0.5, M=1.1, G r=2, \Omega=0.9$, $\operatorname{Pr}=2, R n=0.5, S r=0.6, S c=0.5, G c=1$.
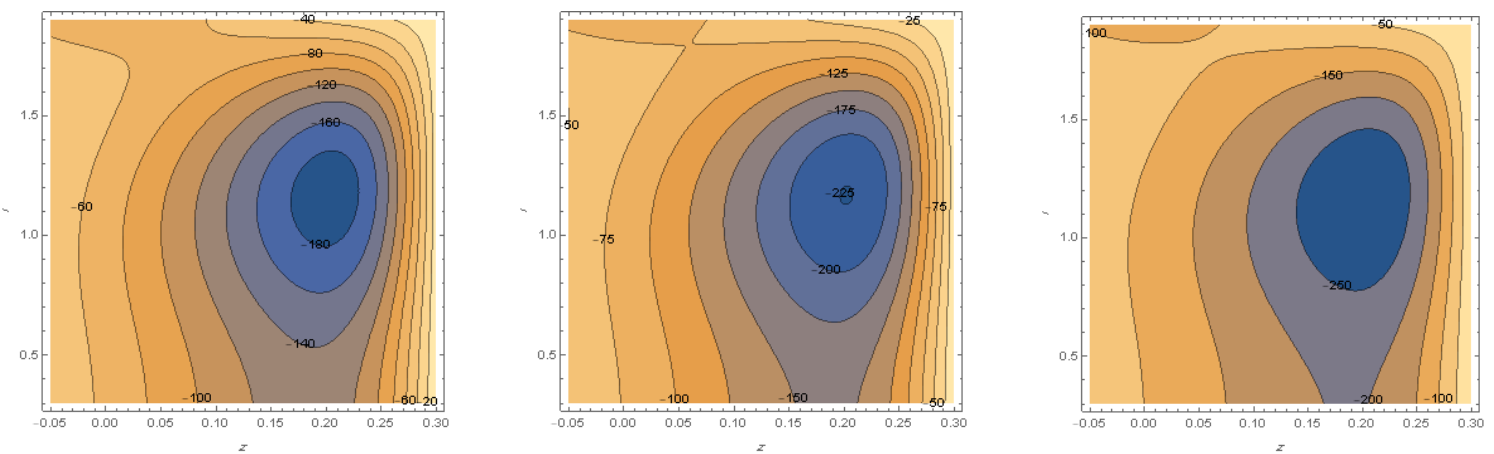

Figure 42: Wave frame streamlines for different values of $L_{1}=\{0.125,0.15,0.175\}$ at $\varepsilon=0.2, \phi=0.2$, $\lambda_{1}=0.1, L_{5}=0.1, L_{4}=0.5, L_{3}=0.1, t=0.05, L_{2}=0.5, \mathrm{Da}=0.9, \alpha=3.75, \zeta=0.5, M=1.1, G r=$ $2, \Omega=0.9, \operatorname{Pr}=2, R n=0.5, S r=0.6, S c=0.5, G c=1$. 

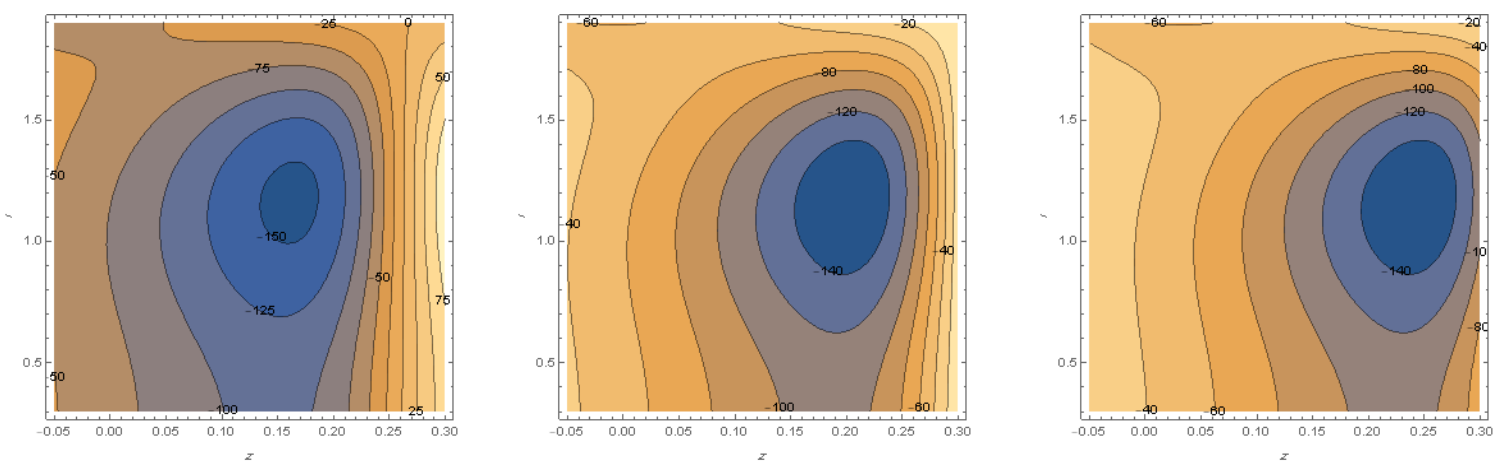

Figure 43: Wave frame streamlines for different values of $t=\{0.01,0.05,0.07\}$ at $\varepsilon=0.2, \phi=0.2, \lambda_{1}=0.1$, $L_{5}=0.1, L_{4}=0.5, L_{3}=0.1, L_{1}=0.1, L_{2}=0.5, \mathrm{Da}=0.9, \alpha=3.75, \zeta=0.5, M=1.1, G r=2, \Omega=0.9$, $\operatorname{Pr}=2, R n=0.5, S r=0.6, S c=0.5, G c=1$.
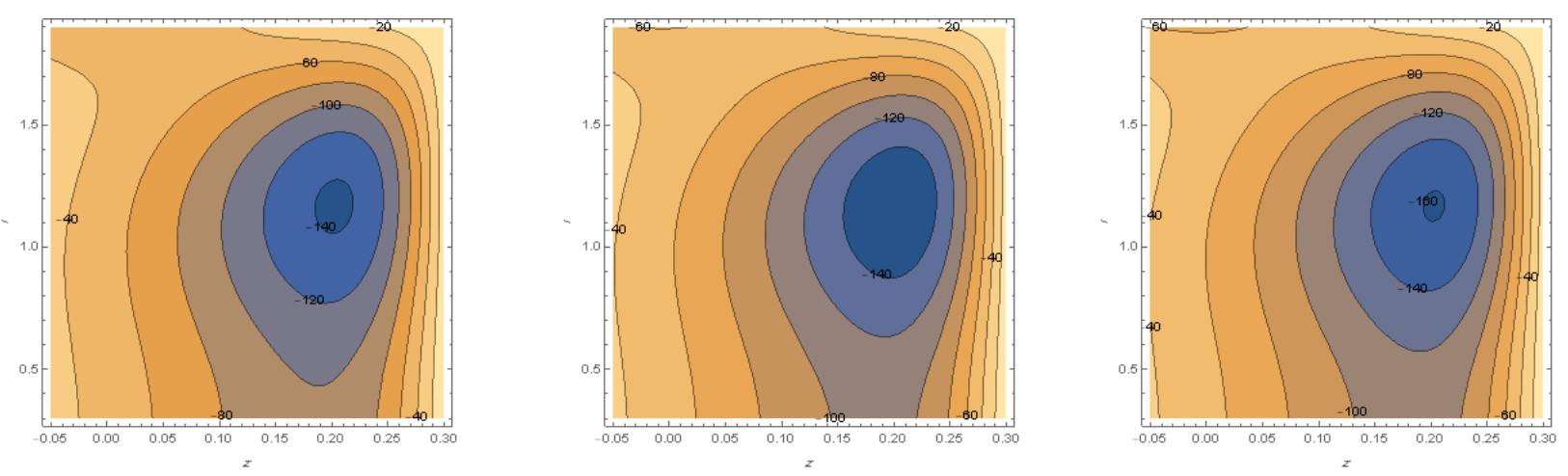

Figure 44: Wave frame streamlines for different values of $L_{2}=\{0.1,0.475,0.625\}$ at $\varepsilon=0.2, \phi=0.2$, $\lambda_{1}=0.1, L_{5}=0.1, \quad L_{4}=0.5, L_{3}=0.1, L_{1}=0.1, t=0.05, \mathrm{Da}=0.9, \alpha=3.75, \zeta=0.5, M=1.1, G r=$ $2, \Omega=0.9, \operatorname{Pr}=2, R n=0.5, S r=0.6, S c=0.5, G c=1$.
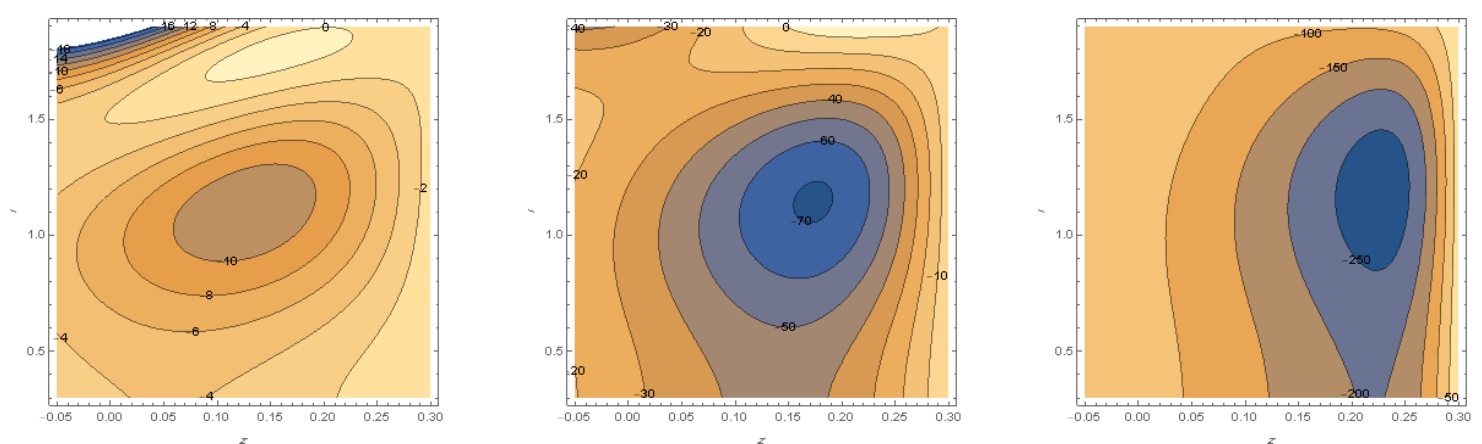

Figure 45: Wave frame streamlines for different values of $D a=\{0.1,0.5,0.9\}$ at $\varepsilon=0.2, \phi=0.2, \lambda_{1}=0.1$, $L_{5}=0.1, L_{4}=0.5, L_{31}=0.1, L_{1}=0.1, t=0.05, L_{2}=0.5, \alpha=3.75, \zeta=0.5, M=1.1, G r=2, \Omega=0.9$, $\operatorname{Pr}=2, R n=0.5, S r=0.6, S c=0.5, G c=1$. 

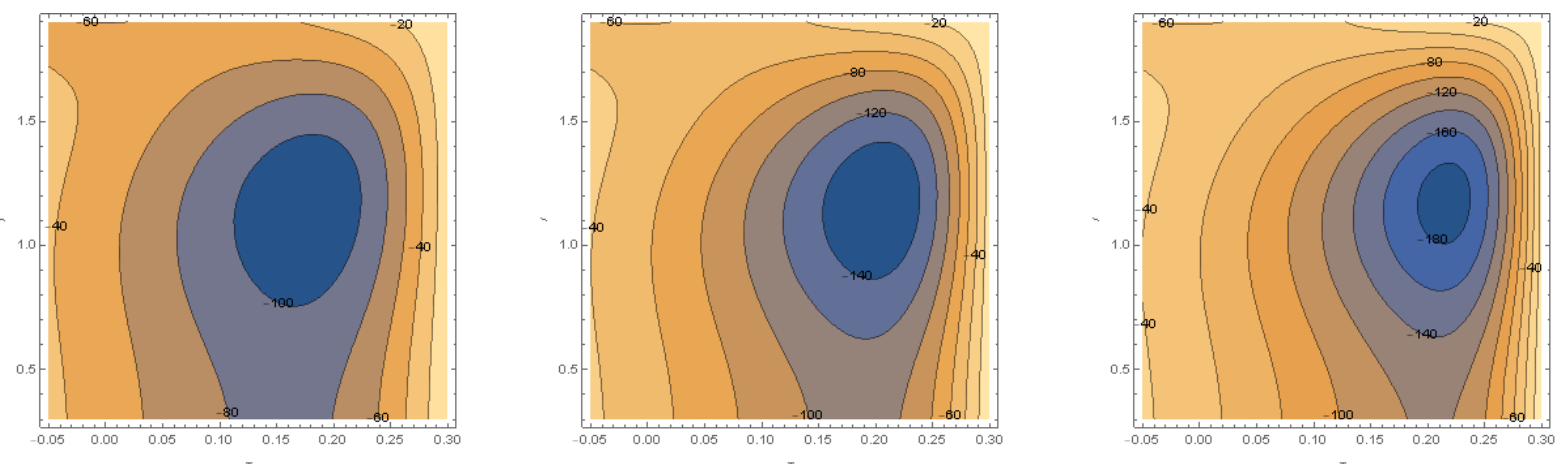

Figure 46: Wave frame streamlines for different values of $\alpha=\{3.65,3.75,3.8\}$ at $\varepsilon=0.2, \phi=0.2, \lambda_{1}=0.1$, $L_{5}=0.1, L_{4}=0.5, L_{3}=0.1, L_{1}=0.1, t=0.05, L_{1}=0.5, D a=0.9, \zeta=0.5, M=1.1, G r=2, \Omega=0.9$, $\operatorname{Pr}=2, R n=0.5, S r=0.6, S c=0.5, G c=1$.
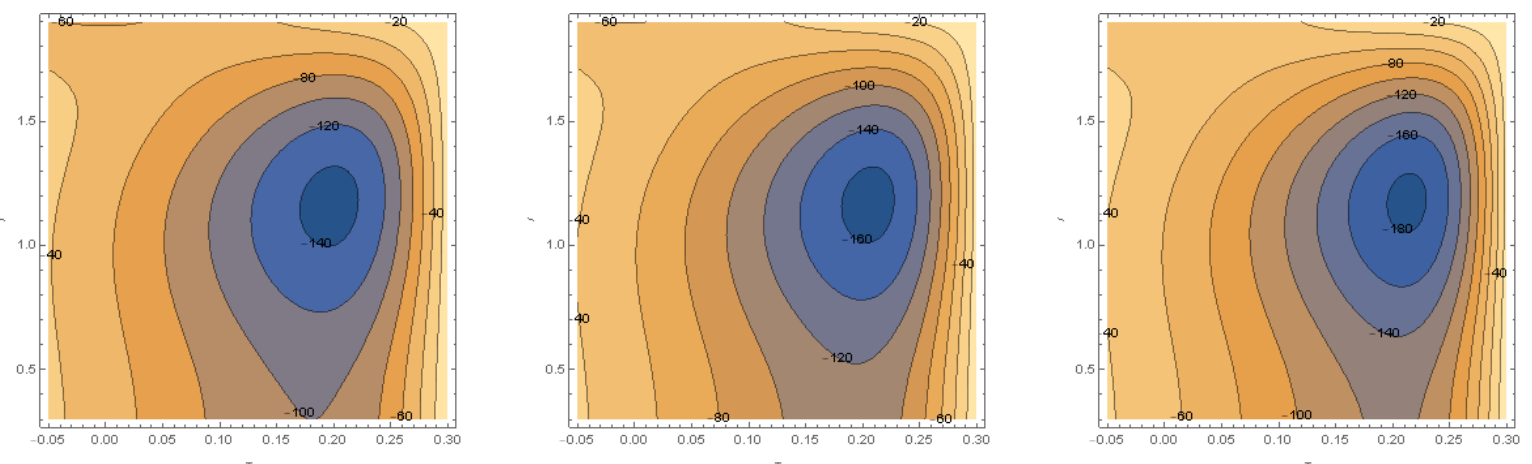

Figure 47: Wave frame streamlines for different values of $\zeta=\{0.425,0.525,0.625\}$ at $\varepsilon=0.2, \phi=0.2$, $\lambda_{1}=0.1, L_{5}=0.1, L_{4}=0.5, L_{3}=0.1, L_{1}=0.1, t=0.05, L_{2}=0.5, D a=0.9, \alpha=3.75, M=1.1, G r=$ $2, \Omega=0.9, \operatorname{Pr}=2, R n=0.5, S r=0.6, S c=0.5, G c=1$.
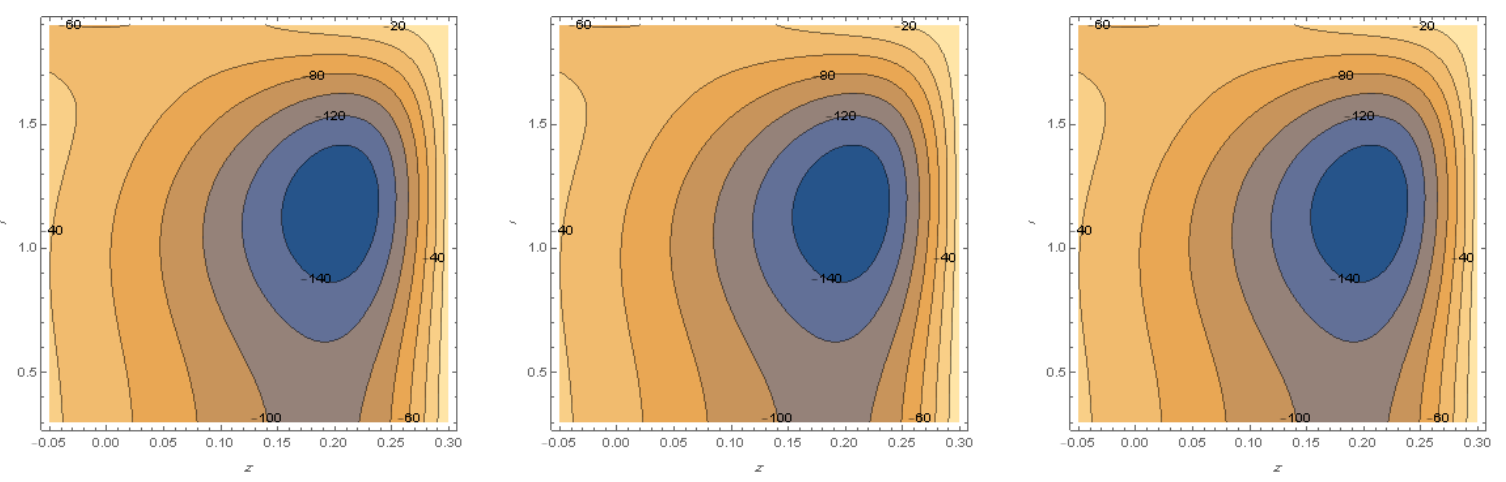

Figure 48: Wave frame streamlines for different values of $M=\{1.1,1.2,1.3\}$ at $\varepsilon=0.2, \phi=0.2, \lambda_{1}=0.1$, $L_{5}=0.1, \quad L_{4}=0.5, L_{3}=0.1, L_{1}=0.1, t=0.05, L_{2}=0.5, D a=0.9, \alpha=3.75, \zeta=0.5, G r=2, \Omega=0.9$, $\operatorname{Pr}=2, R n=0.5, S r=0.6, S c=0.5, G c=1$.

\section{Remarks:}

Important results were found by studying the effects of wall properties on the peristaltic flow of a couple-stress for Jeffrey fluid. Having solved the problem using the Bessel functions, we used the MATHEMATICA program to discuss the effect of efferent parameters on fluid motion by analyzing the obtained graphs. Here is a summary of the results we obtained

- The temperature of fluid rising with an increase of $\Omega, \varepsilon, R n, \operatorname{Pr}$ and $t$, while going down with the increasing $\phi$.

- The Concentration of fluid rising with an increase of $\Omega, \varepsilon, S r, S c, R n, \operatorname{Pr}$ and $t$, while going down with the increasing $\phi$. 
- The velocity of fluid rise up by increasing of $\emptyset, L_{5}, L_{4}, L_{1}, L_{2}, D a$, and $\alpha$, when $r<0.2$, while go down otherwise. The velocity of fluid go down by increasing of $\lambda_{1}$, $L_{3}, t, M, G r, G c$ and $R n$ when $r<0.2$, while rise up otherwise. The velocity of fluid go down by increasing of $\zeta, \Omega, P r, S r$ and $S c$.

- The sizeof the trappedbolus increases with the increasing $\emptyset, L_{5}, L_{4}, L_{1}, L_{2}, D a, \alpha$ and $\zeta$ in the middle of the channel and the bolus will turn into a wave.

- The sizeof the trapped bolusdecreases with the increasing $\varepsilon, \lambda_{1}, L_{3}, M$ and $t$ in the middle of the channel and the wave will turn into a bolus.

\section{References}

[1] Dheia G Salih Al-Khafajy And Ahmed A Hussien Al-Aridhee,. (2019, September). Influence of MHD Peristaltic Transport for Jeffrey Fluid with Varying Temperature and Concentration through Porous Medium. In Journal of Physics: Conference Series (Vol. 1294, No. 3, p. 032012). IOP Publishing.

[2] Dheia G Salih Al-Khafajy,. (2017). Influence of MHD and Wall Properties on the Peristaltic Transport of a Williamson Fluid with Variable Viscosity Through Porous Medium. Iraqi Journal of Science, 58(2C), 1076-1089.

[3] Dheia G. Salih Al-khafajy and Ahmed Abd Alhadi, 2014. Magnetohydrodynamic Peristaltic flow of a couple stress with heat and mass transfer of a Jeffery fluid in a tube through porousmedium, Advances inPhysics Theories andApplications, Vol.32, ISSN 22250638 .

[4] Dheia Gaze Salih Al-Khafajy, Radiation and Mass Transfer Effects on MHD Oscillatory Flow For Jeffery Fluid With Variable Viscosity Through Porous Channel in the Presence of Chemical Reaction, 2019, Sci. Int. (Lahore), 31(2),223-228.

[5] Gramer K.R. and pai-shih-1. 1973. Magnetohydrodynamic for Engineers and Applied physics, McGraw-Hill Book Company, New York.

[6] Hoseinzadeh S, Heyns PS, Chamkha AJ, Shirkhani A. Thermal analysis of porous fins enclosure with the comparison of analytical and numerical methods. J Therm Anal Calorim 2019;138(1).

[7] Latham TW, MS Thesis, M III Cambridge, Massachusetts Institute of Technology, Cambridge, 1966.

[8] M.A. Imran, Fizza Miraj, I. Khan, and Tlili, I. (2018). MHD fractional Jeffrey's fluid flow in the presence of thermo diffusion, thermal radiation effects with first order chemical reaction and uniform heat flux. Results in Physics, 10, 10-17.

[9] M.M. Bhatti, A. Zeeshan, N. Ijazb, O. Anwar Bég and A. Kadir. (2017). Mathematical modelling of nonlinear thermal radiation effects on EMHD peristaltic pumping of viscoelastic dusty fluid through a porous medium duct. Engineering science and technology, an international journal, 20(3), 1129-1139.

[10] Manton, M. J. (1975). Long-wavelength peristaltic pumping at low Reynolds number. Journal of Fluid Mechanics, 68(3), 467-476.

[11] S. Uddin, M. Mohamad, Mohammad Rahimi-Gorji, R. Roslan and Ibrahim M. Alarifi. (2020). Fractional electro-magneto transport of blood modeled with magnetic particles in cylindrical tube without singular kernel. Microsystem Technologies, 26(2), 405-414. 